Preprint typeset in JHEP style - HYPER VERSION

FNT/T 2006/08

IFUM-874/FT

\title{
Precision electroweak calculation of the charged current Drell-Yan process
}

\author{
C.M. Carloni Calame \\ INFN, Sezione di Pavia and Dipartimento di Fisica Nucleare e Teorica, Università di \\ Pavia, Via A.Bassi 6, I-27100 Pavia, Italy \\ Email: Carlo.Carloni.Calame@pv.infn.it
}

\section{G. Montagna}

Dipartimento di Fisica Nucleare e Teorica, Università di Pavia and INFN, Sezione di

Pavia, Via A.Bassi 6, I-27100 Pavia, Italy

Email: Guido.Montagna@pv.infn.it

\section{O. Nicrosini}

INFN, Sezione di Pavia, Via A.Bassi 6, I-27100 Pavia, Italy

Email: Oreste.Nicrosini@pv.infn.it

\section{A. Vicini \\ Dipartimento di Fisica, Università degli Studi di Milano and INFN, Sezione di Milano, Via Celoria 16, I-20133 Milano, Italy \\ Email: Alessandro.Vicini@mi.infn.it}

\begin{abstract}
We present a detailed study of the charged current Drell-Yan process, which includes the exact $\mathcal{O}(\alpha)$ electroweak corrections properly matched with leading-log effects due to multiple-photon emission, as required by the experiments at the Tevatron and the LHC. Numerical results for the relevant observables of single $W$ boson production at hadron colliders are presented. The impact of the radiative corrections and of some sources of theoretical uncertainty is discussed in detail. The calculation has been implemented in the new version of the event generator HORACE, which is available for precision simulations of the charged current Drell-Yan process.
\end{abstract}

KEYWORDs: Drell-Yan, electroweak radiative corrections, QED Parton Shower, matching. 


\section{Contents}

1. Introduction

2. Partonic process: matrix elements calculation 3

2.1 Born approximation 3

2.2 The $\mathcal{O}(\alpha)$ calculation

2.2.1 Virtual corrections

2.2.2 Bremsstrahlung corrections

3. Higher-order corrections and matching procedure 8

4. Hadron-level cross section 10

5. Numerical results 12

5.1 Technical checks 14

$5.2 W$ transverse mass distribution

5.3 Rapidity distributions and charge asymmetry 21

$5.4 W$ transverse momentum and photonic observables 23

6. Conclusions 24

\section{Introduction}

At hadron colliders, such as the Fermilab Tevatron and the CERN LHC, the production of a high transverse momentum lepton pair, known as Drell-Yan process [1], plays an important role: it allows, in the charged current channel, a high precision determination of two fundamental parameters of the Standard Model, namely the mass and the decay width of the $W$ boson [2]; it provides, both in neutral and charged current channels, stringent constraints on the density functions which describe the partonic content of the proton [3]; it can be used as a standard reference process and therefore as a luminosity monitor of the collider [由, 5]. Furthermore, it represents a background to the search for new heavy gauge bosons [6].

The accuracy in the determination of the theoretical cross section has greatly increased over the years. The calculation of next-to-leading order (NLO) QCD corrections [7] has been one of the first test grounds of perturbative QCD. Next-to-next-to-leading order (NNLO) QCD corrections to the total cross section have been computed in ref. [8], but differential distributions with the same accuracy have been obtained only recently in ref. [9]. The size of the NNLO QCD corrections and the improved stability of the results against 
changes of the renormalization/factorization scales raises the question of the relevance of the $\mathcal{O}(\alpha)$ electroweak (EW) radiative corrections, which were computed, in the charged current channel, first in the pole approximation [10, 11] and then fully in refs. [12, 13, 14, 15.

A realistic phenomenological study and the data analysis require the inclusion of the relevant radiative corrections and their implementation into Monte Carlo event generators, in order to simulate all the experimental cuts and to allow, for instance, an accurate determination of the detector acceptances. The Drell-Yan processes are included in the standard QCD Parton Shower generators HERWIG and PYTHIA [16, 17]. Recently there have been important progresses to improve the QCD radiation description to NLO, which has been implemented in the code MC@NLO [18]. Another important issue is the good description of the intrinsic transverse momentum of the gauge boson, which can be obtained by resumming up to all orders the contributions of the form $\alpha_{s} \log \left(p_{\perp}^{W} / m_{W}\right)$. The generator RESBOS [19], used for data analysis at Tevatron, includes these effects.

If the inclusion of QCD radiation is mandatory for the simulation of any process at a hadron collider, one should not neglect the impact of EW corrections on the precision measurement of some Standard Model (SM) observables, like the $W$ boson mass and decay width. For instance, the generator WGRAD [14] includes the exact $\mathcal{O}(\alpha)$ EW corrections, which have been shown to induce a shift on the value of $m_{W}$ extracted from the Tevatron data of about $160 \mathrm{MeV}$ in the muon channel [2], mostly due to final-state QED radiation. In view of the very high experimental precision foreseen at the LHC $\left(\Delta m_{W} \approx 15 \mathrm{MeV}\right)$, final-state higher-order (beyond $\mathcal{O}(\alpha)$ ) QED corrections may induce a significant shift, as shown in ref. [20]. Some event generators can account also for multiple-photon radiation: in the published version of HORACE [20, 21] final-state QED radiation was simulated by means of a QED Parton Shower [22]; the generator WINHAC [23] uses the Yennie-Frautchi-Suura 24] formalism to exponentiate final-state-like $\mathrm{EW} \mathcal{O}(\alpha)$ corrections; finally, the standard tool PHOTOS 25] can be used to describe QED radiation in the $W$ decay.

A first attempt to study the combined effect of EW and QCD corrections has been presented in ref. 26.

The predictions of WINHAC and HORACE and of WINHAC and PHOTOS, for $W$-decay, have been compared in the papers of ref. [27]. A detailed series of tuned comparisons between different EW Monte Carlo generators have been done in ref. [28], in order to check the reliability of different numerical predictions, with $\mathcal{O}(\alpha)$ accuracy, in a given setup of input parameters and cuts.

Since the Drell-Yan events can be used, in principle, to determine the collider luminosity at a few per cent level, the theoretical cross section must be known with the same accuracy, requiring also the inclusion of $\mathcal{O}(\alpha)$ EW corrections. Furthermore, the $\mathcal{O}(\alpha)$ EW contributions give large corrections to the tails of the transverse mass and lepton tranverse momentum distributions, because of the presence of large EW Sudakov logarithms [13, 14]. These regions are important for the search of new heavy gauge bosons.

The aim of this paper is to present a precision EW calculation of the charged current Drell-Yan process, which includes the exact $\mathcal{O}(\alpha)$ EW matrix elements properly matched with leading-logarithmic higher-order QED corrections in the Parton Shower approach. 
The matching here presented of perturbative corrections with Parton Shower, which is a topic of great interest in modern QCD simulations [29], is the first example of such an application in the EW sector and is realized along the lines already presented in ref. [30]. Several distributions of physical interest are analyzed, disentangling the effect of different classes of radiative corrections and discussing various sources of theoretical uncertainty. The calculation is implemented in the Monte Carlo event generator HORACE, which combines, in a unique tool, the good features of the QED Parton Shower approach with the additional effects present in the exact $\mathcal{O}(\alpha) \mathrm{EW}$ calculation. This task is non trivial from several technical points of view and faces all the conceptual problems of developing a NLO event generator.

The paper is organised as follows. In Section 2 we present the calculation of the $\mathcal{O}(\alpha)$ EW corrections to the partonic process $u \bar{d} \rightarrow l^{+} \nu_{l}(\gamma)$. In Section 3 we describe the matching of the fixed order results with the QED Parton Shower. In Section 1 we present the computation of the hadron-level cross section $\sigma\left(p \stackrel{(-)}{p} \rightarrow l^{+} \nu_{l}(n \gamma)\right)$ and discuss the subtraction of the initial-state collinear singularities to all orders. In Section 5 we present phenomenological results for several physical distributions and discuss the impact of EW $\mathcal{O}(\alpha)$ and of higher-order QED corrections. Finally, in Section 6 we draw our conclusion and discuss possible developments of this work.

\section{Partonic process: matrix elements calculation}

\subsection{Born approximation}

We consider the charged current Drell-Yan partonic process $u\left(p_{1}\right) \bar{d}\left(p_{2}\right) \rightarrow \nu_{l}\left(p_{3}\right) l^{+}\left(p_{4}\right)$. This process is a weak charged current process and its amplitude, in unitary gauge, is proportional, at tree level, to the square of the $S U(2)_{L}$ coupling constant $g$ :

$$
\mathcal{M}_{0}=i \frac{g^{2} V_{u d}}{2} \frac{g_{\mu \nu}-k_{\mu} k_{\nu} / m_{W}^{2}}{s-m_{W}^{2}+i \Gamma_{W} m_{W}}\left[\bar{v}\left(p_{2}\right) \gamma^{\mu} \frac{1-\gamma_{5}}{2} u\left(p_{1}\right)\right]\left[\bar{u}\left(p_{3}\right) \gamma^{\nu} \frac{1-\gamma_{5}}{2} v\left(p_{4}\right)\right]
$$

where $V_{u d}$ is the CKM matrix-element, $m_{W}$ is the $W$-boson mass and $\Gamma_{W}$ is the $W$ decay width, necessary to describe the $W$ resonance region, $s=\left(p_{1}+p_{2}\right)^{2}$ is the squared centerof-mass energy and $k^{\mu}=p_{1}^{\mu}+p_{2}^{\mu}$. The differential cross section, mediated over initial-state and summed over final-state spins, mediated over initial-state colours and in the limit of vanishing fermion masses, is given by

$$
\frac{d \sigma_{0}}{d \Omega}=\frac{g^{4}\left|V_{u d}\right|^{2}}{768 \pi^{2}} \frac{1}{\left(s-m_{W}^{2}\right)^{2}+\Gamma_{W}^{2} m_{W}^{2}} \frac{u^{2}}{s}
$$

where $u=\left(p_{1}-p_{4}\right)^{2}$. The weak coupling $g$ can be expressed in terms of $\alpha$, the fine structure constant, and of $\sin \theta_{W}$, the sinus of the weak mixing angle, via the relation $4 \pi \alpha=g^{2} \sin ^{2} \theta_{W}$. The weak mixing angle is defined as $\cos \theta_{W} \equiv m_{W} / m_{Z}$, where $m_{Z}$ is the $Z$ boson mass. 


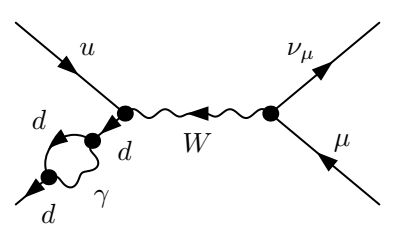

(a)

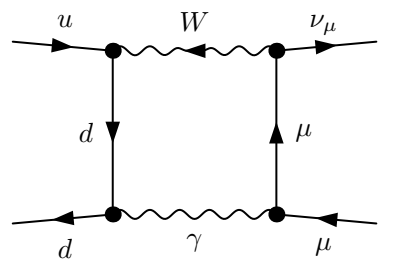

(d)

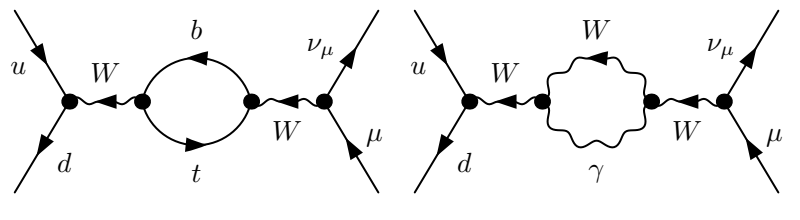

(b)

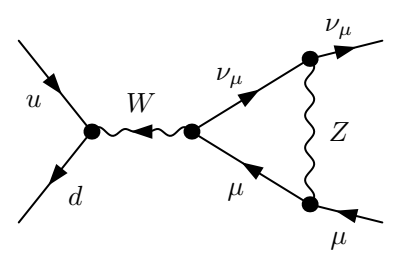

(e) (c)

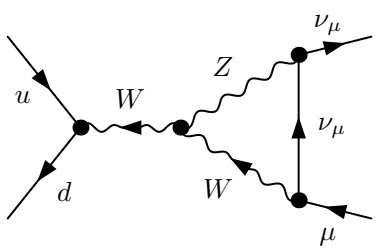

(f)

Figure 1: Some examples of one-loop virtual diagrams.

\subsection{The $\mathcal{O}(\alpha)$ calculation}

The complete EW $\mathcal{O}(\alpha)$ corrections to the charged current Drell-Yan process have already been computed in refs. 110, 11, 12, 13, 14, 15]. We have repeated independently the calculation and we summarize here its main features.

The $\mathcal{O}(\alpha)$ corrections include the contribution of real and virtual corrections. The virtual corrections follow from the perturbative expansion of the $2 \rightarrow 2$ scattering amplitude $\mathcal{M}=\mathcal{M}_{0}+\mathcal{M}_{\alpha}^{\text {virt }}+\cdots$ and contribute, at $\mathcal{O}(\alpha)$, with $2 \operatorname{Re}\left(\mathcal{M}_{\alpha}^{\text {virt }} \mathcal{M}_{0}^{*}\right)$. The $\mathcal{O}(\alpha)$ virtual amplitude includes two contributions, namely the one-loop renormalization of the treelevel amplitude and the virtual one-loop diagrams. The real corrections are due to the emission of one extra real photon and represent the lowest order of the radiative process $u\left(p_{1}\right) \bar{d}\left(p_{2}\right) \rightarrow \nu_{l}\left(p_{3}\right) l^{+}\left(p_{4}\right) \gamma(k)$. They can be further divided in soft and hard corrections, $\mathcal{M}_{1}=\mathcal{M}_{1}^{\text {soft }}+\mathcal{M}_{1}^{\text {hard }}$. The former respect, by definition, the Born-like $2 \rightarrow 2$ kinematics and can be factorized as $\left|\mathcal{M}_{1}^{\text {soft }}\right|^{2}=\delta_{S B}\left|\mathcal{M}_{0}\right|^{2}$ where $\delta_{S B}$ is a universal factor that depends only on the external particles. The total cross section includes soft and hard corrections and is independent of the cut-off used to define the two energy regions. Virtual and real soft corrections are separately divergent due to the emission of soft photons, but the divergence cancels in the sum of the two contributions.

\subsubsection{Virtual corrections}

The $\mathcal{O}(\alpha)$ virtual corrections to a $2 \rightarrow 2$ reaction include contributions of counterterm, selfenergy, vertex and box corrections. Few diagrams representative of the different kinds of corrections are depicted in figure 11 and have been calculated using the packages FeynArts and FormCalc [31, 32]. The numerical evaluation of the 1-loop integrals has been done using the package LoopTools2 [32], based on the library ff [33]. We will write the 1loop virtual amplitude as $\mathcal{M}_{\alpha}^{\text {virt }}=\mathcal{M}_{\alpha}^{\text {cts }}+\mathcal{M}_{\alpha}^{\text {self }}+\mathcal{M}_{\alpha}^{\text {vertex }}+\mathcal{M}_{\alpha}^{\text {box }}$. We have explicitly 
checked that our results numerically agree with those of refs. [13, 15]; the fermion mass corrections, which are present in our calculation and neglected in refs. [13, 15], turn out to be negligible from a numerical point of view. The mass of the fermions in the scalar 1-loop integrals regularizes in a natural way the mass singularities due to the emission of a (virtual) collinear photon. The infrared divergence of the integrals has been regularized by means of a small photon mass $\lambda$.

The introduction of the $W$ decay width in the propagator of the $W$ boson is mandatory to describe the resonance region and to regularize the divergence due to the pole of the propagator. In order to account for the $W$ width, we observe that the $W$ propagator, in the 't Hooft-Feynman gauge, can be modified at 1-loop in the following way:

$$
\begin{aligned}
& \left(-i g^{\mu \nu}\right) \frac{1}{s-m_{W}^{2}+i \Gamma_{W} m_{W}} \rightarrow \\
& \left(-i g^{\mu \nu}\right) \frac{1}{s-m_{W}^{2}+i \Gamma_{W} m_{W}}\left(\Pi_{W W}(s)+\delta m_{W}^{2}+\left(s-m_{W}^{2}\right) \delta Z_{W}\right) \frac{1}{s-m_{W}^{2}}
\end{aligned}
$$

where $\Pi_{W W}(s)$ is the transverse part of the $W$ self-energy corrections, $\delta m_{W}^{2}$ and $\delta Z_{W}$ are respectively the $W$ mass and wave function renormalization constants. The two counterterms cancel the divergences present in the self-energy corrections. We remark that the second factor $1 /\left(s-m_{W}^{2}\right)$ in eq. (2.3) is not corrected by the decay width, to avoid double counting; we can check, by expanding the self-energy corrections about $s=m_{W}^{2}$, that the $\mathcal{O}(\alpha)$ expression is regular for $s \rightarrow m_{W}^{2}$. The contemporary presence of the decay width in the propagator and of the explicit $\mathcal{O}(\alpha)$ corrections does not yield any double counting [13]: in fact the imaginary part of the self-energy corrections contributes only at the 2-loop level to the cross section and does not enter in the 1-loop virtual contribution $2 \operatorname{Re}\left(\mathcal{M}_{\alpha}^{\text {virt }} \mathcal{M}_{0}^{*}\right)$. The $W$ self-energy includes also contributions from diagrams having a $W$ and a photon running in the loop, which develop a logarithmic divergence when $s=m_{W}^{2}$. In the 1-loop virtual amplitude, the coefficient of $\log \left(s-m_{W}^{2}\right)$ is gauge invariant; we can therefore regularize the logarithmic divergence by replacing $\log \left(s-m_{W}^{2}\right) \rightarrow \log \left(s-m_{W}^{2}+i \Gamma_{W} m_{W}\right)$ in all the self-energy, vertex and box scalar integrals, without spoiling the gauge-invariance of the calculation.

Examples of vertex and box corrections are depicted in figure 1. The vertex diagrams with a trilinear gauge boson vertex and the box diagrams with a photon in the loop yield the logarithmic divergence at $s=m_{W}^{2}$ previously discussed. The abelian vertex diagrams and the box diagrams with a photonic correction are infra-red divergent. All the vertex and box diagrams with a $Z$ boson exchange yield the so-called EW Sudakov logarithms, namely terms like $\alpha \log ^{2}\left(s / m_{z}^{2}\right)$, whose importance grows for large invariant mass of the final state lepton pair, while they are almost negligible at the $W$ resonance.

The calculation has been repeated with two different gauge choices, namely the $R_{\xi}$ with $\xi=1$ gauge and the background field gauge, with parameter $q=1$ [34]. The two results perfectly agree, and this is an important check on the calculation of the bosonic self-energy and of the non-abelian vertex corrections.

Concerning the renormalization of the 1-loop amplitude, the UV divergences which appear from the virtual diagrams can be cancelled with the mass, $\delta m_{W}^{2}$, and wave func- 
tion, $\delta Z_{W}$, renormalization constants of the $W$ boson and by the renormalization of the two vertices $W u \bar{d}$ and $W l^{+} \nu_{l}$. The latter include the charge renormalization and the wave function renormalization of the external fermions and of the $W$ boson. Being the tree-level amplitude of eq. (2.1) proportional to $g_{0}^{2}$, using the electric charge and the gauge boson masses $\left(e, m_{W}, m_{Z}\right)$ as input parameters, we write the bare coupling $g_{0}=e_{0} / s_{W, 0}$ and then replace it in terms of renormalized quantities and of counterterms $g_{0}=e / s_{W}(1-\delta e / e) /(1-$ $\left.\delta s_{W} / s_{W}\right)$. The electric charge counterterm is fixed by the request that in Thomson scattering the renormalized charge is given by the fine structure constant; its expression depends on the quark masses running in the photon vacuum polarization, the value of which can be adjusted in order to make the running electric charge reproduce the value $\alpha\left(m_{z}^{2}\right)$ [35]. The weak mixing angle counterterm is given as a combination of the mass counterterms of the $W$ and $Z$ bosons, following from its definition. The $W$ boson mass and wave function renormalization constants are defined in the on-shell scheme.

The choice of the input parameters of the SM lagrangian has an impact on the prediction of the physical observables. If ideally one were able to resum exactly the perturbative expansion, the predictions would be the same in any scheme. On the other hand the truncation of the perturbative expansion induces a dependence on the scheme, which is formally of higher order, but which can be numerically relevant.

Some of the possible options in the gauge sector of the EW SM are: i) $\alpha, G_{\mu}, m_{Z}$, ii) $\alpha, m_{W}, m_{Z}$, iii) $G_{\mu}, m_{W}, m_{Z}$. The first option, used for LEP1 analyses, is based on the best measured EW quantities and minimizes the parametric dependence of the predictions on the inputs. In this input scheme, the value of $m_{W}$ is a predicted quantity. For $W$ physics at hadron colliders, the other schemes are preferable because $m_{W}$ is an input parameter.

The second choice $(\alpha(0)$-scheme) has the proper coupling $\alpha$ for the real photon emission diagrams and parametrizes the charged current coupling $g$ as $\sqrt{4 \pi \alpha} / s_{W}$.

The choice which seems to be more natural for $W$ physics is the third one ( $G_{\mu}$ scheme), where the weak coupling $g$ is related to the Fermi constant and to the $W$ boson mass by the relation

$$
\frac{G_{\mu}}{\sqrt{2}}=\frac{g^{2}}{8 m_{W}^{2}}(1+\Delta r)
$$

The quantity $\Delta r$ represents all the radiative corrections to the muon-decay amplitude [36]. Introducing

$$
g^{2}=4 \sqrt{2} G_{\mu} m_{W}^{2}(1-\Delta r)
$$

in the tree level amplitude (2.1) we generate an additional contribution to the $\mathcal{O}(\alpha)$ correction proportional to $\Delta r$. Being the vertex between charged particles and photons proportional to $g \sin \theta_{W}$, we can therefore introduce an effective electromagnetic coupling constant

$$
\alpha_{G_{\mu}}^{\text {tree }}=\frac{\sqrt{2} G_{\mu} \sin ^{2} \theta_{W} m_{W}^{2}}{\pi}
$$

which is derived from eq. (2.5) and is evaluated in tree-level approximation by setting $\Delta r=0$. The effective coupling $\alpha_{G_{\mu}}$ differs from the fine structure constant $\alpha$, evaluated at zero momentum transfer, by higher-order effects. 


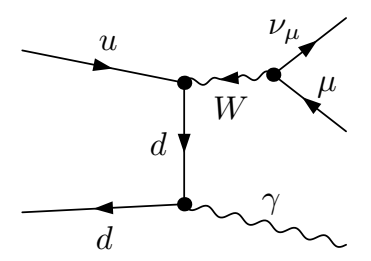

(a)

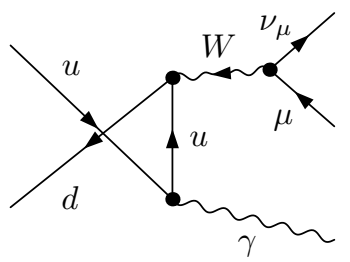

(b)

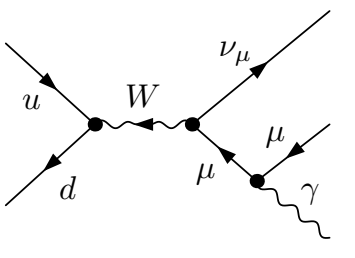

(c)

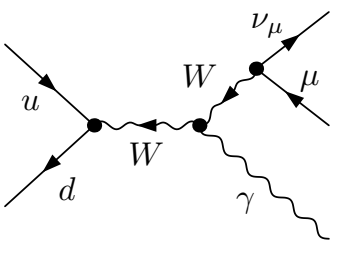

(d)

Figure 2: $\mathcal{O}(\alpha)$ bremsstrahlung diagrams.

\subsubsection{Bremsstrahlung corrections}

The real radiative corrections to the charged Drell-Yan process, described by the amplitude $\mathcal{M}_{1}$, are given by all the Feynman diagrams (figure 2) with the emission of one extra photon, from all the electrically charged legs of the Born diagram, including the internal $W$ boson.

The probability amplitude has been calculated in the unitary gauge with massive fermions. We integrate the squared matrix element over the whole photon phase space and split the allowed photon energy range in two intervals, $[\lambda, \Delta E]$ and $\left[\Delta E, E_{\max }\right]$. The cut-off $\Delta E \ll \sqrt{s}$ is chosen in such a way that the photon with smaller energy is considered soft and does not modify the $2 \rightarrow 2$ kinematics of the Born amplitude. The small photon mass $\lambda$ has been introduced to regularize the infrared divergence. In this energy region the phase space integral, including the full angular integration, can be solved analytically. The result can be expressed in a factorized form, as

$$
\int_{\Omega} \frac{d^{3} \mathbf{k}_{\gamma}}{(2 \pi)^{3} 2 E_{\gamma}}\left|\mathcal{M}_{1}\right|^{2}=\left|\mathcal{M}_{0}\right|^{2} \sum_{f=u, \bar{d}, e^{+}} \delta_{S B}(f, \lambda)
$$

where the soft Bremsstrahlung factor, see e.g. [37], depends on the mass and electric charge of the external radiating particles and the phase-space region $\Omega$ is defined by the request that the photon energy $E_{\gamma}$ satisfies $\lambda \leq E_{\gamma} \leq \Delta E$. We have explicitly checked that the sum of the virtual and soft-real contributions is independent of the choice of the photon mass $\lambda$, in the limit of small $\lambda$ values.

In the hard energy region the phase space integration has been performed numerically, with Monte Carlo techniques improved by importance sampling to take care of collinear and infrared singularities, as well as the peaking behaviour around the $W$ resonance. The sum of the soft and of the hard photon cross sections is independent of the cut-off $\Delta E$. We have checked the independence of our numerical results from the choice of the infrared separator $\varepsilon \equiv \Delta E / E$ for $10^{-8} \leq \varepsilon \leq 10^{-4}$.

The squared matrix element $\left|\mathcal{M}_{1}\right|^{2}$ has been calculated and evaluated with good agreement in several different ways, using FeynArts and FormCalc, by hand with the help of FORM [38], using the ALPHA algorithm [39], in order to check the numerical stability of the results. 


\section{Higher-order corrections and matching procedure}

In this section we describe the matching of the fixed EW $\mathcal{O}(\alpha)$ calculation with higherorder QED corrections (cfr. ref. [30]). The latter can be included in a generic scattering cross section in the QED Parton Shower approach, which resums to all orders the leading logarithmic effects. At $\mathcal{O}(\alpha)$ the Parton Shower reproduces only the QED leading-log approximation of the exact $\mathrm{EW} \mathcal{O}(\alpha)$ calculation, presented in section 2. We would like to combine the exact $\mathcal{O}(\alpha)$ results and QED higher orders, to improve the approximation intrinsic to the Parton Shower, avoiding at the same time double countings at $\mathcal{O}(\alpha)$.

The matching of the two calculations is a non trivial task and we present, for the sake of clarity, the main ideas in a simplified unphysical toy model, namely in the case of a scattering process where only one external particle can radiate. A general expression of its cross section with the emission of an arbitrary number of photons, in leading-log approximation, can be cast in the following way:

$$
d \sigma^{\infty}=\Pi\left(Q^{2}, \varepsilon\right) \sum_{n=0}^{\infty} \frac{1}{n !}\left|\mathcal{M}_{n, L L}\right|^{2} d \Phi_{n}
$$

where $\Pi\left(Q^{2}, \varepsilon\right)$ is the Sudakov form-factor that accounts for soft-photon (up to $\varepsilon$ ) and virtual emissions and $Q^{2}$ is related to the energy scale of the hard scattering process. $\left|\mathcal{M}_{n, L L}\right|^{2}$ is the squared amplitude in leading-log approximation describing the process with the emission of $n$ hard photons, with energy larger than $\varepsilon$ in units of the radiating particle energy. $d \Phi_{n}$ is the exact phase-space element of the process, with $n$ photons in the final state, divided by the incoming flux factor. The cross section $d \sigma^{\infty}$ is independent of the infrared separator $\varepsilon$.

According to the theorems for the factorization of soft and collinear photon emission, the leading part of the squared amplitudes can be written in a factorized form; for example the one-photon emission squared amplitude reads [40]

$$
\left|\mathcal{M}_{1, L L}\right|^{2}=\frac{\alpha}{2 \pi} P(z) I(k) \frac{8 \pi^{2}}{E^{2} z(1-z)}\left|\mathcal{M}_{0}\right|^{2}
$$

where $1-z$ is the fraction of energy carried by the photon, $I(k)$ is a function which describes the angular spectrum of the photon and $P(z)=\left(1+z^{2}\right) /(1-z)$ is the Altarelli-Parisi $e \rightarrow e+\gamma$ splitting function. In eq. (3.2) we observe the factorization of the Born squared amplitude and that the emission factor can be iterated to all orders, giving $\left|\mathcal{M}_{n, L L}\right|^{2}$. The Sudakov form factor $\Pi\left(Q^{2}, \varepsilon\right)$ can be expressed as

$$
\Pi\left(Q^{2}, \varepsilon\right)=\exp \left(-\frac{\alpha}{2 \pi} I_{+} \log \frac{Q^{2}}{m^{2}}\right), \quad I_{+} \equiv \int_{0}^{1-\varepsilon} d z P(z)
$$

The function $I(k)$ has the property that $\int d \Omega_{\gamma} I(k)=\log Q^{2} / m^{2}$ and allows the cancellation of the infrared logarithms.

It would be desirable to include in eq. (3.1) the missing $\mathcal{O}(\alpha)$ contributions. The matching procedure can be better understood by comparing the exact $\mathcal{O}(\alpha)$ cross section 
with the $\mathcal{O}(\alpha)$ expansion of eq. (3.1), which, as we already mentioned, does not coincide, by definition, with an exact $\mathcal{O}(\alpha)$ result. In fact

$$
\begin{aligned}
d \sigma_{\alpha, L L} & =\left[1-\frac{\alpha}{2 \pi} I_{+} \log \frac{Q^{2}}{m^{2}}\right]\left|\mathcal{M}_{0}\right|^{2} d \Phi_{0}+\left|\mathcal{M}_{1, L L}\right|^{2} d \Phi_{1} \\
& \equiv\left[1+C_{\alpha, L L}\right]\left|\mathcal{M}_{0}\right|^{2} d \Phi_{0}+\left|\mathcal{M}_{1, L L}\right|^{2} d \Phi_{1}
\end{aligned}
$$

whereas an exact NLO cross section can be always cast in the form

$$
d \sigma_{\alpha}=\left[1+C_{\alpha}\right]\left|\mathcal{M}_{0}\right|^{2} d \Phi_{0}+\left|\mathcal{M}_{1}\right|^{2} d \Phi_{1}
$$

The coefficient $C_{\alpha}$ contains the complete virtual $\mathcal{O}(\alpha)$ and the $\mathcal{O}(\alpha)$ soft-bremsstrahlung squared matrix elements, in units of the Born squared amplitude and $\left|\mathcal{M}_{1}\right|^{2}$ is the exact squared matrix element with the emission of one hard photon. We remark that $C_{\alpha, L L}$ and $\left|\mathcal{M}_{1, L L}\right|^{2}$ have the same singular logarithmic structure of $C_{\alpha}$ and of $\left|\mathcal{M}_{1}\right|^{2}$.

We observe that, by introducing the correction factors

$$
F_{S V}=1+\left(C_{\alpha}-C_{\alpha, L L}\right), \quad F_{H}=1+\frac{\left|\mathcal{M}_{1}\right|^{2}-\left|\mathcal{M}_{1, L L}\right|^{2}}{\left|\mathcal{M}_{1, L L}\right|^{2}}
$$

the exact $\mathcal{O}(\alpha)$ cross section can be expressed, up to terms of $\mathcal{O}\left(\alpha^{2}\right)$, in terms of its leading-log approximation as

$$
d \sigma_{\alpha}=F_{S V}\left(1+C_{\alpha, L L}\right)\left|\mathcal{M}_{0}\right|^{2} d \Phi_{0}+F_{H}\left|\mathcal{M}_{1, L L}\right|^{2} d \Phi_{1}
$$

Driven by eq. (3.7), we can improve eq. (3.1) by writing the resummed cross section as

$$
d \sigma^{\infty}=F_{S V} \Pi\left(Q^{2}, \varepsilon\right) \sum_{n=0}^{\infty} \frac{1}{n !}\left(\prod_{i=0}^{n} F_{H, i}\right)\left|\mathcal{M}_{n, L L}\right|^{2} d \Phi_{n}
$$

The correction factors $F_{H, i}$ follow from the definition eq. (3.6) for each photon emission. The expansion at $\mathcal{O}(\alpha)$ of eq. (3.8) coincides now with the exact NLO cross section of eq. (3.5). Furthermore, all higher-order leading-log contributions are the same as in eq. (3.1). It is worth noticing that $F_{S V}, F_{H, i}$ are, by construction, infrared safe and free of collinear logarithms.

Alternatively, we remark that one could have improved eq. (3.1) by adding the $\mathcal{O}(\alpha)$ contributions missing in the leading-log approximation. However, we prefer the factorized formulation eq. (3.8) for different reasons. The first is that it allows to include a large class of radiative corrections to all orders beyond the leading logarithmic approximation. In fact, the factorization of the soft and collinear QED corrections is a universal property valid for any hard scattering process; the latter can be computed not only at tree level, but also including infrared safe radiative corrections; this is the case in eq. (3.8), with the factors $F_{S V}$ and $F_{H, i}$. As a consequence, not only the tower of leading terms of the form $\alpha^{n} \log ^{n}\left(s / M^{2}\right)$ but also all the terms of the form $\alpha^{n} \log ^{n-1}\left(s / M^{2}\right) \log \left(s / \Delta E^{2}\right)$ ( $M$ is the mass of the radiating particle) are correctly taken into account by eq. (3.8). Another reason for choosing a factorized formulation is related to the request that the exponentiated cross 
section should go to zero in the limit of vanishing infrared separator $\varepsilon$ : in the addittive formulation the missing infrared finite $\mathcal{O}(\alpha)$ terms would spoil this property.

Equation (3.8) is our master formula for the matching between the exact $\mathcal{O}(\alpha)$ EW calculation and the QED resummed Parton Shower cross section. Its extension to the realistic case, where every charged particle radiates photons, is almost straightforward.

It is useful to present, in the general case, the expression of the function $I(k)$, which describes the leading behaviour of the angular spectrum of the emitted photons.

$$
I(k)=\sum_{i, j} Q_{i} Q_{j} \eta_{i} \eta_{j} \frac{p_{i} \cdot p_{j}}{p_{i} \cdot k p_{j} \cdot k} E_{\gamma}^{2}
$$

where $Q_{l}$ and $p_{l}$ are the electric charge fraction and the momentum of the external fermion $l, \eta_{l}$ is a charge factor equal to +1 for incoming particles or outgoing antiparticles and equal to -1 for incoming antiparticles or outgoing particles, $k$ is the photon momentum and the sum runs over all the external fermions.

In our case the Sudakov form factor is

$$
\Pi\left(p_{i} ; \varepsilon\right)=\exp \left(-\frac{\alpha}{2 \pi} I_{+} \int d \Omega_{\gamma} I(k)\right)
$$

The result of the analytical integration of $I(k)$ is:

$$
\int d \Omega_{\gamma} I(k)=-\sum_{j>i} \sum_{i} \eta_{i} \eta_{j} Q_{i} Q_{j} \frac{2 \tilde{p}_{i} \cdot p_{j}}{\tilde{p}_{i}^{2}-p_{j}^{2}} \log \frac{\tilde{p}_{i}^{2}}{p_{j}^{2}}-\sum_{i} Q_{i}^{2}
$$

where

$$
\tilde{p}_{i}=\beta_{i j} p_{i}
$$

The parameters $\beta_{i j}$ are obtained requiring that $\left(\tilde{p}_{i}-p_{j}\right)^{2}=0$ and $\tilde{p}_{i}^{0}-p_{j}^{0}=\beta_{i j} p_{i}^{0}-p_{j}^{0}>0$.

\section{Hadron-level cross section}

In this section we discuss the hadron-level cross section $\left.\sigma(p p) \rightarrow l \nu_{l}+n \gamma+X\right)$ and the procedure to subtract the initial-state mass singularities in the calculation of the cross section first with $\mathcal{O}(\alpha)$ and then with the $\mathcal{O}(\alpha)$ improved with QED higher-order corrections. This procedure makes the resulting hadron-level cross section independent of the (unphysical) value of the initial-state quark masses.

The radiative corrections are enhanced by large collinear logarithmic (mass singularities) factors, of the form $\alpha /(2 \pi) \log \left(s / m_{f}^{2}\right)$. The initial-state collinear logarithms are universal, i.e. are independent of the hard scattering process, must be factorized out at an energy scale $M$ and can be reabsorbed in the definition of the parton densities which describe the partonic content of the proton, like in all NLO QCD calculations. Since the partonic cross section described in section 2 still contains QED initial-state mass singularities, it is mandatory to implement a subtraction procedure to avoid a double counting when convoluting with the proton parton densities. Recently a new set of PDFs (MRST2004QED) including QED effects has been published [41]. These parton densities are evolved via the 
DGLAP equations including also the QED splitting functions. As discussed in the literature [43, 44], the effect of the QED evolution on the PDFs is at the per mille level, for $x$ and $M^{2}$ values probed by Drell-Yan dynamics at the Tevatron and at the LHC.

The subtraction at $\mathcal{O}(\alpha)$ has been discussed for example in ref. 113 and is obtained by a redefinition of the parton densities. The hadron-level cross section at $\mathcal{O}(\alpha)$ can be written

$$
\begin{aligned}
d \sigma\left(p \vec{p} \rightarrow l \nu_{l}+X\right)= & \sum_{a, b} \int_{0}^{1} d x_{1} d x_{2} \quad q_{a}\left(x_{1}, M^{2}\right) q_{b}\left(x_{2}, M^{2}\right)\left[d \sigma_{0}+d \sigma_{\alpha}\right]- \\
& -\left(\Delta q_{a}\left(x_{1}, M^{2}\right) q_{b}\left(x_{2}, M^{2}\right)+q_{a}\left(x_{1}, M^{2}\right) \Delta q_{b}\left(x_{2}, M^{2}\right)\right) d \sigma_{0}
\end{aligned}
$$

where $a, b$ run over all parton species described by the densities $q_{i}\left(x, M^{2}\right), M$ is the factorization scale, $d \sigma_{0}$ and $d \sigma_{\alpha}$ are the Born and $\mathcal{O}(\alpha)$ partonic cross sections. The $\mathcal{O}(\alpha)$ subtraction term is

$$
\Delta q_{i}\left(x, M^{2}\right)=\int_{z}^{1} q_{i}\left(\frac{x}{z}, M^{2}\right) \frac{\alpha}{2 \pi} Q_{i}^{2}\left[P(z)\left(\log \left(\frac{M^{2}}{m_{i}^{2}}\right)-2 \log (1-z)-1+f(z)\right)\right]_{+} .
$$

$Q_{i}$ and $m_{i}$ are the electric charge fraction and the mass of the quark $i$ and the function $f(z)$ [14] allows to change the subtraction scheme (e.g. DIS or $\overline{M S}$ ). Given the presence in the hadron-level cross section eq. (4.1) of the product of two parton densities, the subtraction procedure in a factorized form could yield terms of $\mathcal{O}\left(\alpha^{2}\right)$ which have been discarded for consistency at $\mathcal{O}(\alpha)$.

It is mandatory to generalize the independence from the value of the quark masses of the $\mathcal{O}(\alpha)$ cross section of eq. (4.1) to the cross section including also QED higher-order corrections. The exponentiation in the first line of eq. (4.1) of $\left[d \sigma_{0}+d \sigma_{\alpha}\right]$, according to the matching algorithm described in section 3, would develop also higher-order initial-state mass singularities, which appear in the form $\alpha^{n} \log ^{n}\left(s / m_{f}^{2}\right) \quad(n>1)$. In order to remove the latter in a systematic way, we prefer to rewrite the partonic cross section in eq. (4.1); we split it in two terms: one free of initial-state mass singularities, $d \tilde{\sigma}_{\alpha}$, which will be then improved with the resummation, and one which contains the singular part at $\mathcal{O}(\alpha), d \sigma_{\alpha}^{s u b}$. We rewrite eq. (4.1) by adding and subtracting the same quantity, namely

$$
d \sigma_{0}+d \sigma_{\alpha} \rightarrow d \sigma_{0}+\left(d \sigma_{\alpha}-d \sigma_{\alpha}^{s u b}\right)+d \sigma_{\alpha}^{s u b} \equiv d \sigma_{0}+d \tilde{\sigma}_{\alpha}+d \sigma_{\alpha}^{s u b}
$$

The subtraction term is further split into a soft+virtual and a hard photon contributions:

$$
\begin{aligned}
d \sigma_{\alpha}^{\text {sub }} & \equiv d \sigma_{\alpha}^{S V, \text { sub }}+d \sigma_{\alpha}^{H, \text { sub }} \\
d \sigma_{\alpha}^{S V, \text { sub }} & =-\frac{\alpha}{2 \pi}\left[Q_{a}^{2}\left(\log \frac{M^{2}}{m_{a}^{2}}-1\right)+Q_{b}^{2}\left(\log \frac{M^{2}}{m_{b}^{2}}-1\right)\right] \cdot I_{+}\left|\mathcal{M}_{0}\right|^{2} d \Phi_{0} \equiv C_{\alpha}^{s u b}\left|\mathcal{M}_{0}\right|^{2} d \Phi_{0} \\
d \sigma_{\alpha}^{H, \text { sub }} & =\frac{8 \pi \alpha}{E^{2} z(1-z)} \frac{1+z^{2}}{1-z}\left|\mathcal{M}_{0}\left(s^{\prime}\right)\right|^{2} I_{\text {sub }}(k) d \Phi_{1} \equiv\left|\mathcal{M}_{1, \text { sub }}\right|^{2} d \Phi_{1} \\
I_{\text {sub }}(k) & =\sum_{i=a, b} Q_{i}^{2}\left(\frac{1}{1-\beta_{i} c}-\frac{2 m_{i}^{2}}{M^{2}\left(1-\beta_{i} c\right)^{2}}\right)
\end{aligned}
$$


where the subtraction cross sections are defined in analogy to the leading log cross sections of eq. (3.4) and $I_{\text {sub }}(k)$ describes the initial-state radiation. In eq. (4.4) $\left|\mathcal{M}_{0}\left(s^{\prime}\right)\right|^{2}$ is the Born squared amplitude evaluated at a reduced center-of-mass energy $s^{\prime}, \beta_{i}=$ $\sqrt{1-4 m_{i}^{2} / M^{2}}$ and $c$ is the cosine of the angle of the photon with the beam axis in the partonic center-of-mass frame. Once integrated over the photonic variables, $d \sigma_{\alpha}^{H, s u b}$ develops the same infrared logarithmic structure as $d \sigma_{\alpha}^{S V, s u b}$ and the sum is independent of the value chosen for the infrared separator.

By applying the matching algorithm to $\left[d \sigma_{0}+d \tilde{\sigma}_{\alpha}\right]$, which is free of collinear initial-state logarithms, we obtain an improved hadron-level cross section including QED higher-order corrections. Starting from the $\mathcal{O}(\alpha)$ expression, identical to eq. 4.1),

$$
\begin{aligned}
d \sigma_{\text {had }} & =\sum_{a, b} \int_{0}^{1} d x_{1} d x_{2} \quad q_{a}\left(x_{1}, M^{2}\right) q_{b}\left(x_{2}, M^{2}\right)\left[d \sigma_{0}+d \tilde{\sigma}_{\alpha}\right]+ \\
& +q_{a}\left(x_{1}, M^{2}\right) q_{b}\left(x_{2}, M^{2}\right)\left[d \sigma_{\alpha}^{s u b}-\left(\frac{\Delta q_{a}\left(x_{1}, M^{2}\right)}{q_{a}\left(x_{1}, M^{2}\right)}+\frac{\Delta q_{b}\left(x_{2}, M^{2}\right)}{q_{b}\left(x_{2}, M^{2}\right)}\right) d \sigma_{0}\right]
\end{aligned}
$$

we obtain the hadron-level QED resummed cross section

$$
\begin{aligned}
& d \sigma_{\text {had }}=\sum_{a, b} \int_{0}^{1} d x_{1} d x_{2} q_{a}\left(x_{1}, M^{2}\right) q_{b}\left(x_{2}, M^{2}\right) \times \\
& \left\{\tilde{F}_{S V} \tilde{\Pi}\left(Q^{2}, \varepsilon\right) \sum_{n=0}^{\infty} \frac{1}{n !}\left(\prod_{i=0}^{n} \tilde{F}_{H, i}\right)\left|\tilde{\mathcal{M}}_{n, L L}\right|^{2} d \Phi_{n}+\right. \\
& \left.+\left[d \sigma_{\alpha}^{s u b}-\left(\frac{\Delta q_{a}\left(x_{1}, M^{2}\right)}{q_{a}\left(x_{1}, M^{2}\right)}+\frac{\Delta q_{b}\left(x_{2}, M^{2}\right)}{q_{b}\left(x_{2}, M^{2}\right)}\right) d \sigma_{0}\right]\right\} .
\end{aligned}
$$

The variables with a tilde represent quantities subtracted of the initial-state singularities; more precisely the matching of section 3 is built using the following subtracted quantities:

$$
\begin{aligned}
& \left|\tilde{\mathcal{M}}_{1}\right|^{2}=\left|\mathcal{M}_{1}\right|^{2}-\left|\mathcal{M}_{1, \text { sub }}\right|^{2}, \quad\left|\tilde{\mathcal{M}}_{1, L L}\right|^{2}=\left|\mathcal{M}_{1, L L}\right|^{2}-\left|\mathcal{M}_{1, \text { sub }}\right|^{2} \\
& \tilde{C}_{\alpha}=C_{\alpha}-C_{\alpha}^{\text {sub }}, \quad \tilde{C}_{\alpha, L L}=C_{\alpha, L L}-C_{\alpha}^{\text {sub }}, \quad \tilde{I}(k)=I(k)-I_{\text {sub }}(k)
\end{aligned}
$$

The $\mathcal{O}(\alpha)$ expansion of eq. (4.6) coincides with eq. (4.5) or equivalently to eq. (4.1). The second line in eq. (4.6) is by construction free of initial-state mass singularities and we remark that the same property holds also for the finite $\mathcal{O}(\alpha)$ correction of the last line.

Equation (4.6) is our master formula for the computation of the hadron-level cross sections and the event simulation.

\section{Numerical results}

All the numerical results have been obtained using the following values for the input parameters: 


$\begin{array}{lll}\alpha=1 / 137.03599911 & G_{\mu}=1.1663710^{-5} \mathrm{GeV}^{-2} & m_{z}=91.1876 \mathrm{GeV} \\ m_{W}=80.425 \mathrm{GeV} & \Gamma_{W}=2.124 \mathrm{GeV} & m_{H}=115 \mathrm{GeV} \\ m_{e}=510.99892 \mathrm{KeV} & m_{\mu}=105.658369 \mathrm{MeV} & m_{\tau}=1.77699 \mathrm{GeV} \\ m_{u}=66 \mathrm{MeV} & m_{c}=1.2 \mathrm{GeV} & m_{t}=178 \mathrm{GeV} \\ m_{d}=66 \mathrm{MeV} & m_{s}=150 \mathrm{MeV} & m_{b}=4.3 \mathrm{MeV} \\ V_{u d}=0.975 & V_{u s}=0.222 & V_{u b}=0 \\ V_{c d}=0.222 & V_{c s}=0.975 & V_{c b}=0 \\ V_{t d}=0 & V_{t s}=0 & V_{t b}=1\end{array}$

and have been computed in the $G_{\mu}$ input scheme described in Section 2. The set of parton density functions used to compute all the hadron-level cross sections is MRST2004QED [41] * . In this set of PDFs the QCD and the QED factorization scales are set to be equal and, as usually done in the literature [13, 14], we choose $M=m_{W}$, if not stated otherwise. The use of the PDFs set MRST2004QED implies that our numerical results correspond to the DIS factorization scheme. The computation of the hadron-level results requires the numerical evaluation of the subtraction term defined in eq. 4.2; a grid of values in the variable $x$, which is then interpolated, is obtained by means of the numerical library CUBA [42]. All the hadron-level results refer to the LHC, at a nominal center-of-mass energy $\sqrt{s}=14 \mathrm{TeV}$, if not stated otherwise.

The fine structure constant $\alpha$ is used instead of $\alpha_{G_{\mu}}^{\text {tree }}$ of eq. (2.6) in the computation of all the $\mathcal{O}(\alpha)$ and higher-order corrections, in order to describe the real photon emission with the proper coupling. This replacement is formally justified in the fixed $\mathcal{O}(\alpha)$ calculation because it differs at $\mathcal{O}\left(\alpha^{2}\right)$. The $G_{\mu}$ input scheme has been implemented by computing all the contributions to the cross section with $\alpha=\alpha(0)$, subtracting the $\Delta r$ contribution, evaluated as well with $\alpha(0)$, and then rescaling the total result by $\left(\alpha_{G_{\mu}}^{\text {tree }} / \alpha(0)\right)^{2}$.

The following cuts have been imposed to select the events:

$$
p_{\perp, \ell}>25 \mathrm{GeV}, \quad p_{\perp, \text { missing }}>25 \mathrm{GeV}, \quad\left|\eta_{\ell}\right|<2.5
$$

where $p_{\perp, \ell}$ and $\eta_{\ell}$ are the transverse momentum and the pseudo-rapidity of the charged lepton and $p_{\perp, \text { missing }}$ is the missing transverse momentum, which in our case coincides with the neutrino $p_{\perp}$.

Our results are obtained with bare muons and with "recombined" electrons. We assume perfect isolation of photons from the muon, which is experimentally achievable with good accuracy: the resulting cross sections are therefore enhanced by large muon mass collinear logarithms, because the photon emission is not treated inclusively in the region about the muon. In the case of electrons, it is experimentally difficult to separate them from the photon track, when the latter lyes within a cone around the lepton. We adopt the following recombination procedure:

- photons with a rapidity $\left|\eta_{\gamma}\right|>2.5$ are never recombined to the electron;

${ }^{*}$ Since this set of PDFs includes a photon distribution function resulting from the QED evolution of the PDFs, the Drell-Yan cross section receives a new type of correction from $2 \rightarrow 3$ photon-induced processes, such as, for example, $\gamma q \rightarrow q^{\prime} l \nu_{l}$. These contributions, which have been evaluated by Dittmaier and Krämer in ref. [28], are not considered in the present study. 


\begin{tabular}{|l|l|}
\hline 1. & lowest order (Born) \\
2. & final state $\mathcal{O}(\alpha)$ LL QED Parton Shower \\
3. & final state exponentiated LL QED Parton Shower \\
4. & exact $\mathcal{O}(\alpha)$ EW of eq. (4.1) \\
5. & exact $\mathcal{O}(\alpha)$ EW matched with higher-order QED corrections (best) of eq. (4.6) \\
\hline
\end{tabular}

Table 1: Different approximations for the cross section of the Drell-Yan charged current process.

\begin{tabular}{|c|c|c|}
\hline$\varepsilon$ & $\mathcal{O}(\alpha)$ & best \\
\hline $5 \cdot 10^{-4}$ & $4410.98 \pm 0.20$ & $4412.14 \pm 0.26$ \\
\hline $1 \cdot 10^{-6}$ & $4410.84 \pm 0.48$ & $4413.66 \pm 0.9$ \\
\hline
\end{tabular}

Table 2: Independence of the hadron-level cross section (pb) of the value chosen for the infrared separator $\varepsilon$.

- if the photon rapidity is $\left|\eta_{\gamma}\right|<2.5$ and $R_{e \gamma}=\sqrt{\left(\eta_{e}-\eta_{\gamma}\right)^{2}+\phi_{e \gamma}^{2}}<0.1\left(\phi_{e \gamma}\right.$ is the angle between the photon and the electron in the transverse plane), then the photon is recombined with the electron, i.e. the momenta of the two particles are added and associated with the momentum of the electron;

- the resulting momenta should satisfy the cuts of eq. (5.1).

In order to study the different effects of the radiative corrections on the relevant observables, we will distinguish the approximations described in table 1. The approximations 2. and 3. refer to the old version of HORACE [20], which included the final-state QED corrections in leading-log accuracy (both at $\mathcal{O}(\alpha)$ and with higher orders) in a pure Parton Shower approach.

\subsection{Technical checks}

In this section we discuss some technical checks satisfied by the new version of HORACE, namely the independence from the $\varepsilon$ parameter and from the quark masses. As reference, we also provide the (un-subtracted) cross section at parton level for three different centerof-mass energies.

The definition of the soft- and hard-bremsstrahlung corrections requires the introduction of an infrared separator $\varepsilon$. The physical cross section does not depend on this parameter as shown in table 目.

The partonic cross section is computed using quark masses to regularize the initialstate collinear singularities. The latter are subtracted according to the procedure described in Section 1 when calculating the hadron-level cross section, which has to be independent, up to terms of order $m_{q}^{2} / m_{W}^{2}$, of the value chosen for the quark masses. This property is demonstrated in table 3, both for the $\mathcal{O}(\alpha)$ and matched cross section. 


\begin{tabular}{|c|c|c|}
\hline & $\mathcal{O}(\alpha)$ & best \\
\hline$m_{q}$ & $4410.98 \pm 0.20$ & $4412.14 \pm 0.26$ \\
\hline$m_{q} / 10$ & $4410.92 \pm 0.26$ & $4411.89 \pm 0.33$ \\
\hline$m_{q} / 100$ & $4410.99 \pm 0.29$ & $4411.92 \pm 0.50$ \\
\hline
\end{tabular}

Table 3: Indipendence of the hadron-level $\mathcal{O}(\alpha)$ and exponentiated cross sections (pb) of the value chosen for the quark masses.

\begin{tabular}{|c|c|c|c|}
\hline$\sqrt{s}(\mathrm{GeV})$ & 60 & 80 & 100 \\
\hline Born & $7.807 \pm 0.001$ & $5223.0 \pm 0.5$ & $25.452 \pm 0.002$ \\
\hline $\mathcal{O}(\alpha)$ & $7.274 \pm 0.002$ & $4817.1 \pm 0.8$ & $34.476 \pm 0.005$ \\
\hline best & $7.288 \pm 0.002$ & $4830.6 \pm 0.6$ & $34.208 \pm 0.008$ \\
\hline
\end{tabular}

Table 4: Unsubtracted partonic cross section (pb) at different partonic center-of-mass energies.

Finally, the partonic cross section has been computed, within the cuts of eq. (5.1), without subtraction of the initial-state collinear divergences; the results are presented in table 1 .

\section{$5.2 W$ transverse mass distribution}

We present in this section the numerical results for the distribution of $W$ transverse mass, defined as

$$
M_{\perp}=\sqrt{2 p_{\perp, \ell} p_{\perp, \nu}\left(1-\cos \phi_{\ell, \nu}\right)}
$$

where $\phi_{\ell, \nu}$ is the angle between the lepton and the neutrino in the transverse plane. We discuss in detail the effect of the different classes of radiative corrections. Their impact, relative to the Born approximation, is in general of several per mille and in some cases of few per cent and can play a significant role for instance in the precise determination of the mass of the $W$ boson $m_{W}$, foreseen at the LHC with an accuracy of $15 \mathrm{MeV}$.

In table 5 we show the cross section integrated within the cuts eq. (5.1) and a futher cut on the minimum $W$ transverse mass, varying from 50 to $2000 \mathrm{GeV}$. The relative effect of the $\mathcal{O}(\alpha)$ corrections grows with the cut on the minimum transverse mass, because of the increasing importance of the EW Sudakov logs.

The Born results coincide for muons and electrons, up to negligible mass effects. The radiative corrections instead differ because of the final-state collinear logarithmic enhancement, which are absent in the case of photons recombined with the electron. All the QED higher-order corrections do not modify significantly the $\mathcal{O}(\alpha)$ corrections.

In figures from 3 to 7 we show the transverse mass distribution and disentangle the different contributions due to the radiative corrections. In figure 3 the transverse mass distribution is plotted, in the range $50<M_{\perp}<100 \mathrm{GeV}$. The transverse mass distribution provides physical information in different ranges: the position of the jacobian peak and the shape of the distribution about the peak can be used to extract the value of the $W$ boson mass, the shape of the tail of the distribution above the peak, $80<M_{\perp}<100$ 


\begin{tabular}{|c|c|c|c|c|c|}
\hline$m_{\perp, \min }(\mathrm{GeV})$ & Born $(\mathrm{pb})$ & $\delta_{\alpha}^{\mu^{+}}(\%)$ & $\delta_{\infty}^{\mu^{+}}(\%)$ & $\delta_{\alpha}^{e^{+}}(\%)$ & $\delta_{\infty}^{e^{+}}(\%)$ \\
\hline 50 & $4536.03(7)$ & -2.8 & -2.7 & -1.7 & -1.8 \\
\hline 100 & $27.642(1)$ & -5.0 & -4.9 & -3.4 & -3.4 \\
\hline 200 & $1.79275(5)$ & -7.9 & -7.7 & -6.3 & -6.3 \\
\hline 500 & $0.084809(2)$ & -14.3 & -13.8 & -12.2 & -12.2 \\
\hline 1000 & $0.0065320(2)$ & -21.9 & -21.1 & -19.4 & -19.1 \\
\hline 2000 & $0.000273686(8)$ & -32.1 & -30.5 & -28.7 & -28.1 \\
\hline
\end{tabular}

Table 5: Lowest-order hadron-level cross section, integrated imposing a cut on the minimum transverse mass and relative effects, with respect to the Born cross section, in the $\mathcal{O}(\alpha)\left(\delta_{\alpha}^{\ell}\right)$ and in the best $\left(\delta_{\infty}^{\ell}\right)$ approximations.

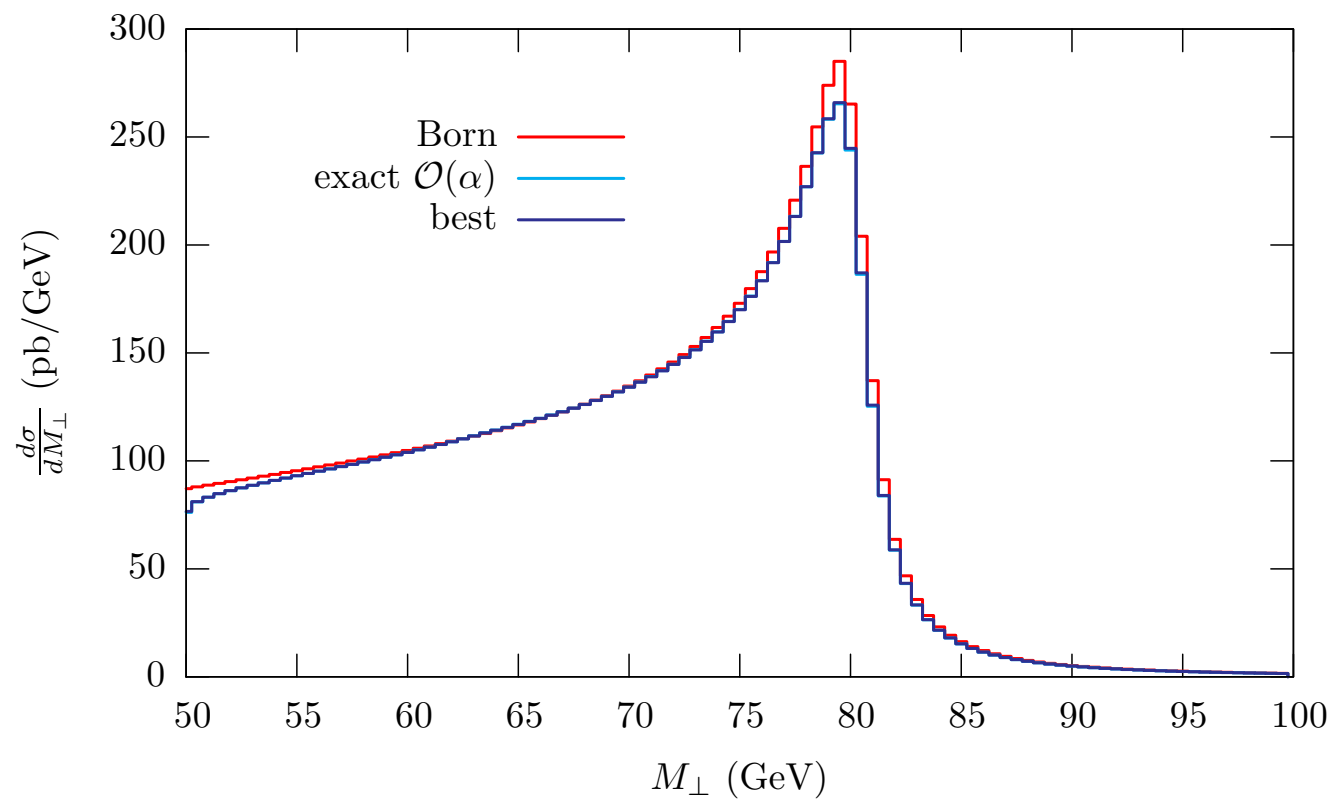

Figure 3: Transverse mass distribution in Born, $\mathcal{O}(\alpha)$ and best approximations.

$\mathrm{GeV}$, can be used to measure the $W$ boson decay width and the large transverse mass tail, $200<M_{\perp}<1000 \mathrm{GeV}$, of the distribution can be an important background to the searches of new heavy gauge bosons.

In figure 1 we plot, in the range $50<M_{\perp}<100 \mathrm{GeV}$ the effect of the exact $\mathcal{O}(\alpha)$ radiative correction, relative to the Born cross section, in the case of muons and of recombined electrons. The $\mathcal{O}(\alpha)$ contribution gives a large correction, up to $\sim-10 \%$, which distorts, about the $W$ resonance, the transverse mass distribution and is responsible for the bulk of the shift in the extraction of the $W$ boson mass. As shown in figure 5 , in the range $100<M_{\perp}<1000 \mathrm{GeV}$ the $\mathrm{EW}$ Sudakov logarithms make the effect of the radiative corrections large and negative, reaching the $20 \%$ level.

In figure 6 we disentangle, among the $\mathcal{O}(\alpha)$ contributions, the effect of all the corrections which can not be classified as QED final state-like leading-log radiation, by taking 


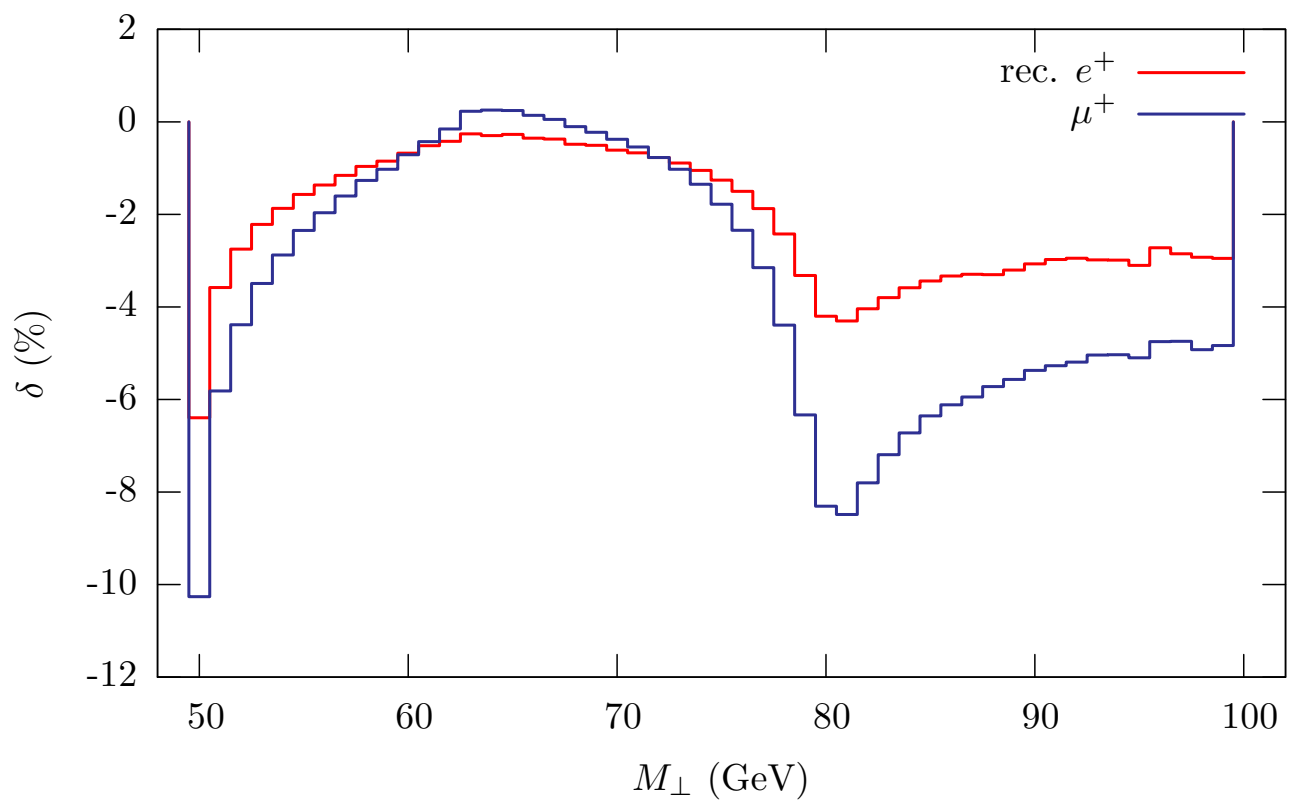

Figure 4: Relative corrections with respect to the Born cross section due to the exact $\mathcal{O}(\alpha)$ corrections for muons and recombined electrons final states.

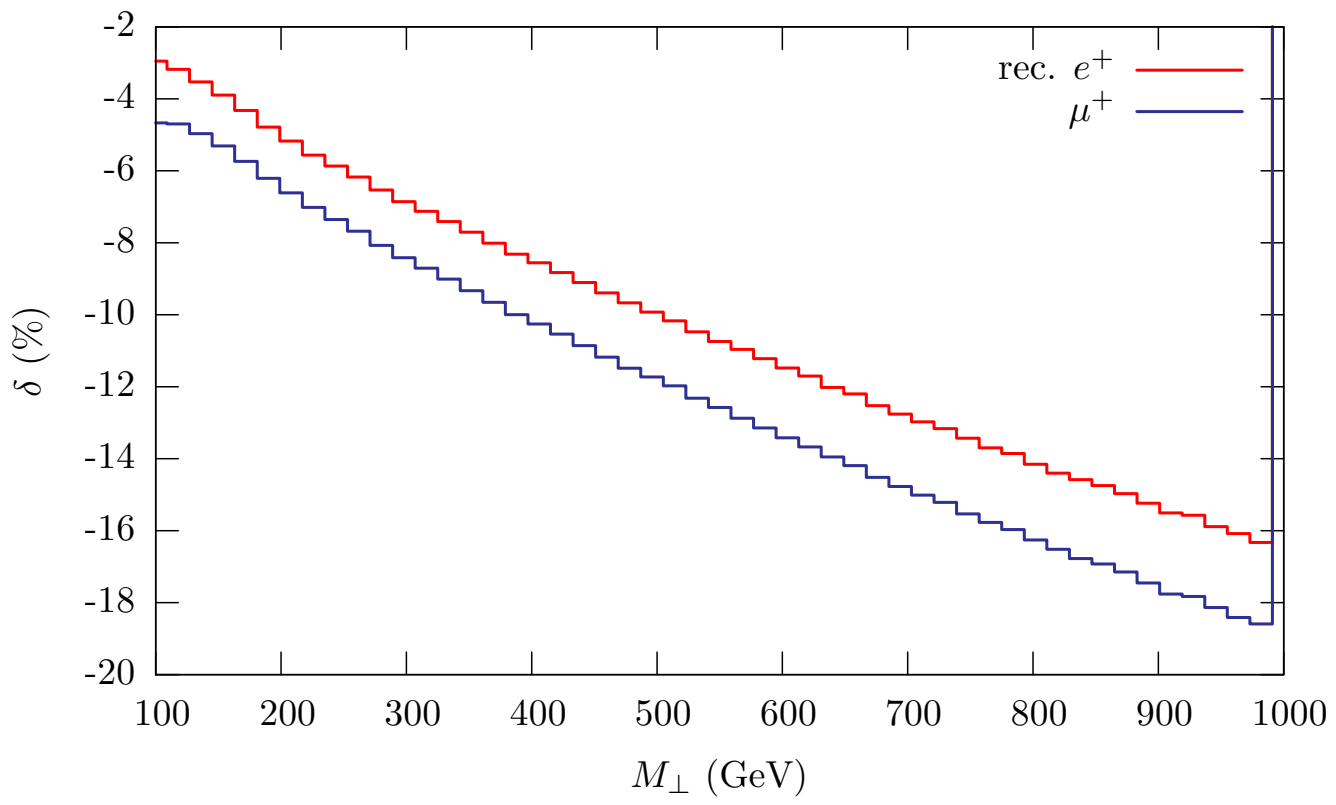

Figure 5: Relative corrections with respect to the Born cross section due to the exact $\mathcal{O}(\alpha)$ corrections for muons and recombined electrons final states.

(blue line) the difference between approximations 4. and 2. (and between 5. and 3., red line) of table 1 in units of the differential Born cross section. We present only the results for muons, being the effect similar in the electron case. We observe that they are quite 

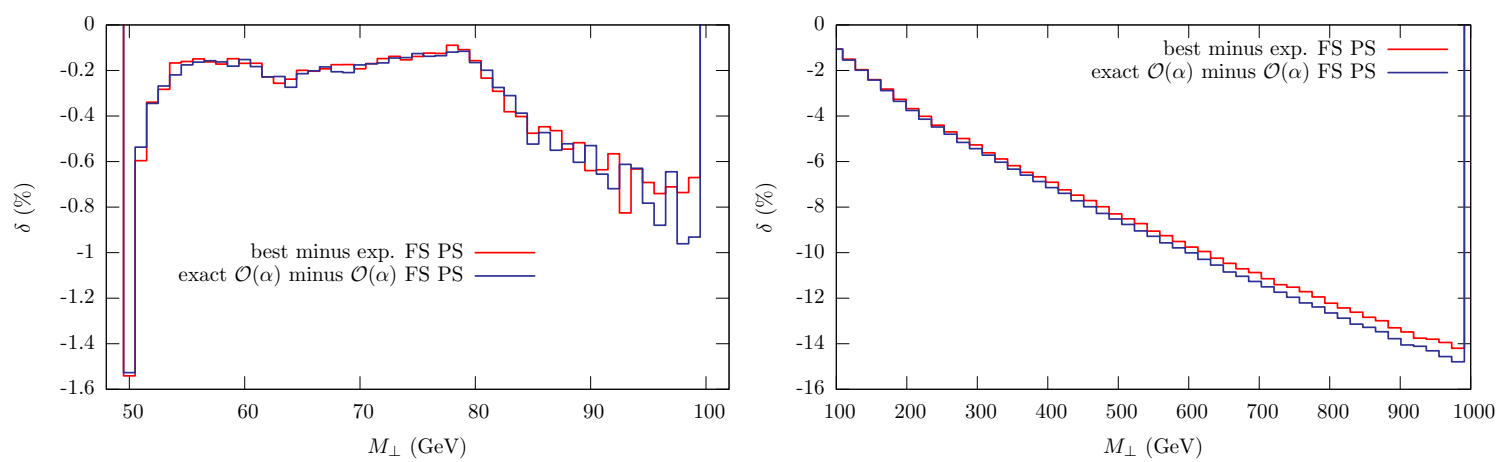

Figure 6: Relative effect, in Born units, of the difference between the approximations 4. and 2. of table 1 (blue line) and between 5. and 3. (red line).
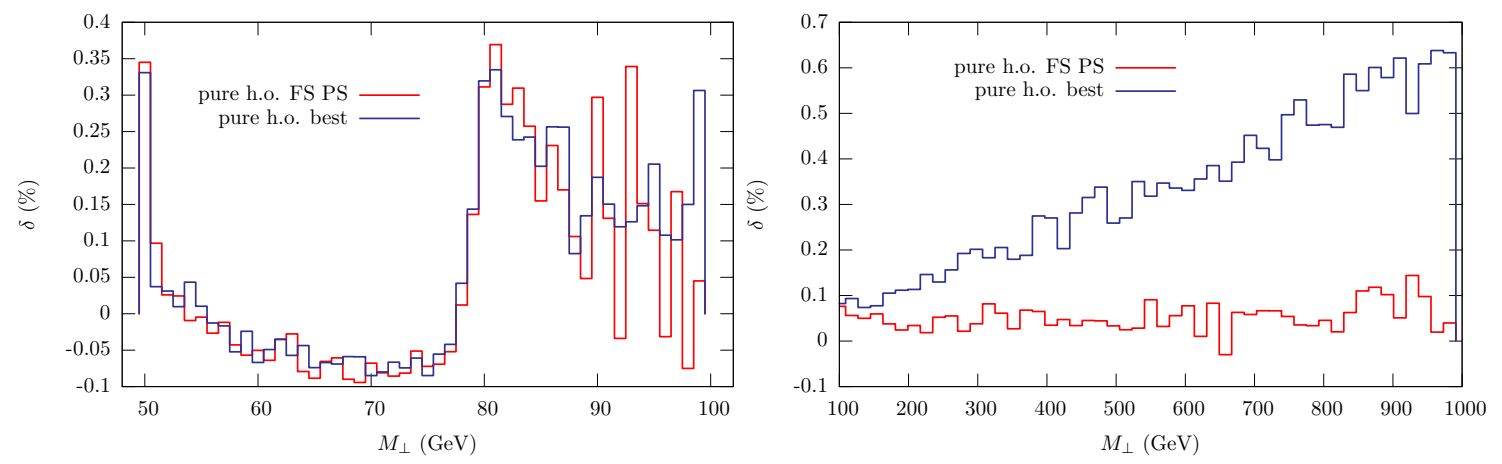

Figure 7: Relative effect on the transverse mass distribution, in Born units, of higher-order QED final state-like and full QED parton shower corrections.

flat, small and negative, for $M_{\perp}<80 \mathrm{GeV}$; they become larger in size and always negative for increasing values of $M_{\perp}$, because of the presence of the EW Sudakov logs. From a comparison of figures 5 and 6 , the non-factorizable weak contributions account for more than half of the $\mathcal{O}(\alpha)$ radiative corrections, for $M_{\perp}>200 \mathrm{GeV}$.

In figure 7 we present the effect of the higher-order (beyond $\mathcal{O}(\alpha)$ ) corrections, and disentangle the effect of all the terms which can not be classified as QED final state-like leading log radiation, by considering the difference of the 3 . and 2. (red line) and of 5 . and 4. (blue line) approximations, in units of the lowest-order differential cross section. We present only the results for muons, being the higher-order corrections smaller in the electron case because of the recombination. The red line describes the effect of purely photonic final-state like leading log corrections, whereas the blue line represents the higherorder contributions of the matched cross section of eq. (4.6). The latter includes, besides the content of the red line, the remnant of the initial-state radiation after the subtraction of the initial-state singularities and the product of purely weak corrections (the $\tilde{F}_{S V}$ factor of eq. (4.6)) with photonic radiation. Around the peak the two lines almost coincide, while for large $M_{\perp}$ we observe the effect of the product of the EW Sudakov logs times the $\mathcal{O}(\alpha)$ photonic correction. 


\begin{tabular}{|c|c|c|c|}
\hline scheme & Born & $\mathcal{O}(\alpha)$ & $\delta(\%)$ \\
\hline$\alpha(0)$ & $4244.68 \pm 0.09$ & $4360.5 \pm 0.6$ & +2.73 \\
\hline$G_{\mu}$ & $4536.03 \pm 0.07$ & $4411.0 \pm 0.2$ & -2.76 \\
\hline
\end{tabular}

Table 6: Born and $\mathcal{O}(\alpha)$ hadron-level cross sections (pb)and effect of the $\mathcal{O}(\alpha)$ corrections, expressed in units of the corresponding Born cross section, in the $\alpha(0)$ and in the $G_{\mu}$ schemes.

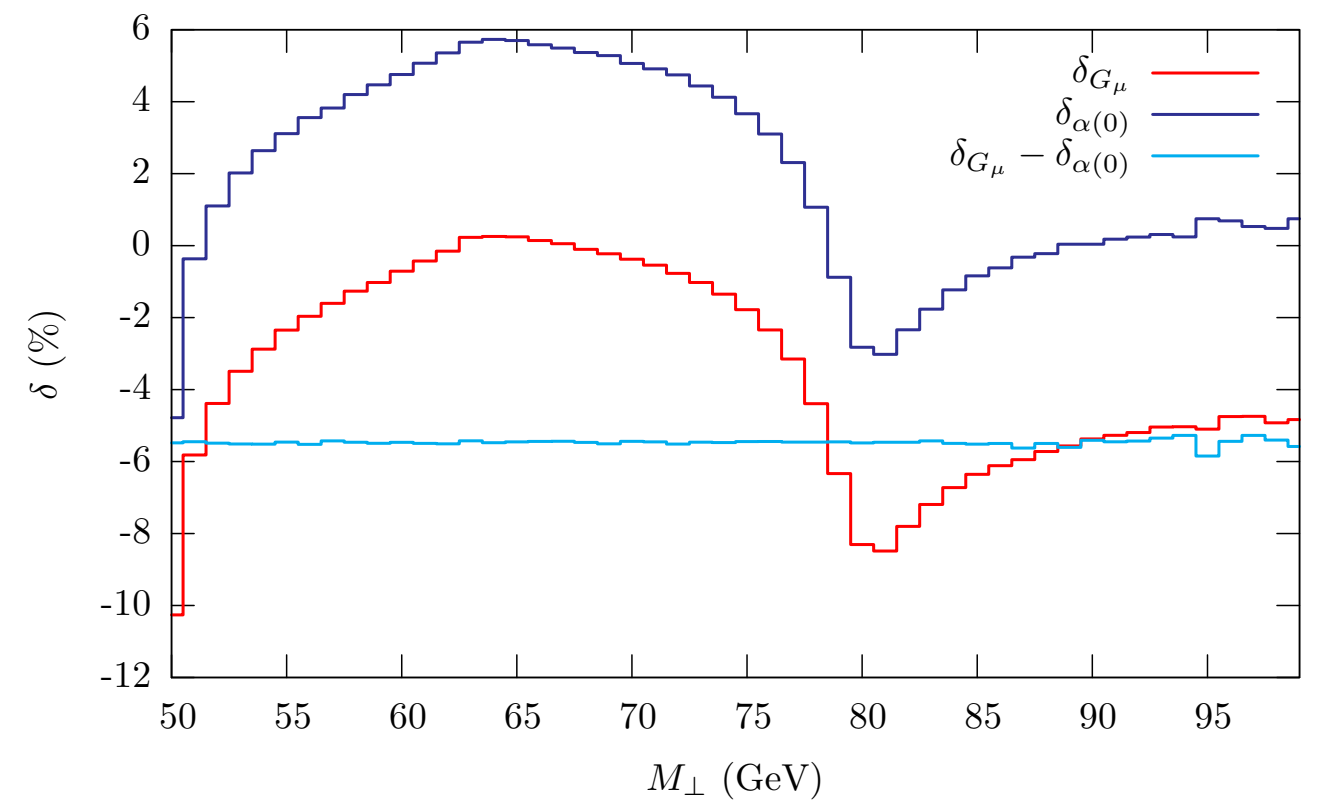

Figure 8: Relative corrections to the transverse mass distribution in the $G_{\mu}$ and in the $\alpha(0)$ schemes, expressed in units of the corresponding Born distributions, and their difference.

As we already discussed in Section 2, we can compute the cross sections in the $G_{\mu}$ or the $\alpha(0)$ input scheme. In table 6, we compare the cross sections obtained in the two input schemes, in Born and in $\mathcal{O}(\alpha)$ approximations and the corresponding relative corrections. The difference between the cross sections in the two schemes is reduced when going from the Born to the $\mathcal{O}(\alpha)$ approximation and amounts to about 6\% (Born) and $1 \%(\mathcal{O}(\alpha))$, respectively. The relative correction in the two schemes is of the same order $(\approx 3 \%)$ but of opposite sign. This can be understood taking into account that, as previously discussed, in the $G_{\mu}$ scheme, at a variance with the $\alpha(0)$ scheme, universal virtual corrections are absorbed in the lowest-order cross section. It is worth noticing that the $\mathcal{O}(\alpha)$ corrected transverse mass distribution differs in the two input schemes as shown in figure 8, where we plot the relative corrections in the two schemes in units of the corresponding Born distributions and their difference.

Another source of uncertainty, which is not of purely EW origin, is the choice in the parton densities of the factorization scale $M$. In order to study this dependence, we set $M=\xi m_{W}$ and consider the canonical range $1 / 2 \leq \xi \leq 2$. We define the two following 


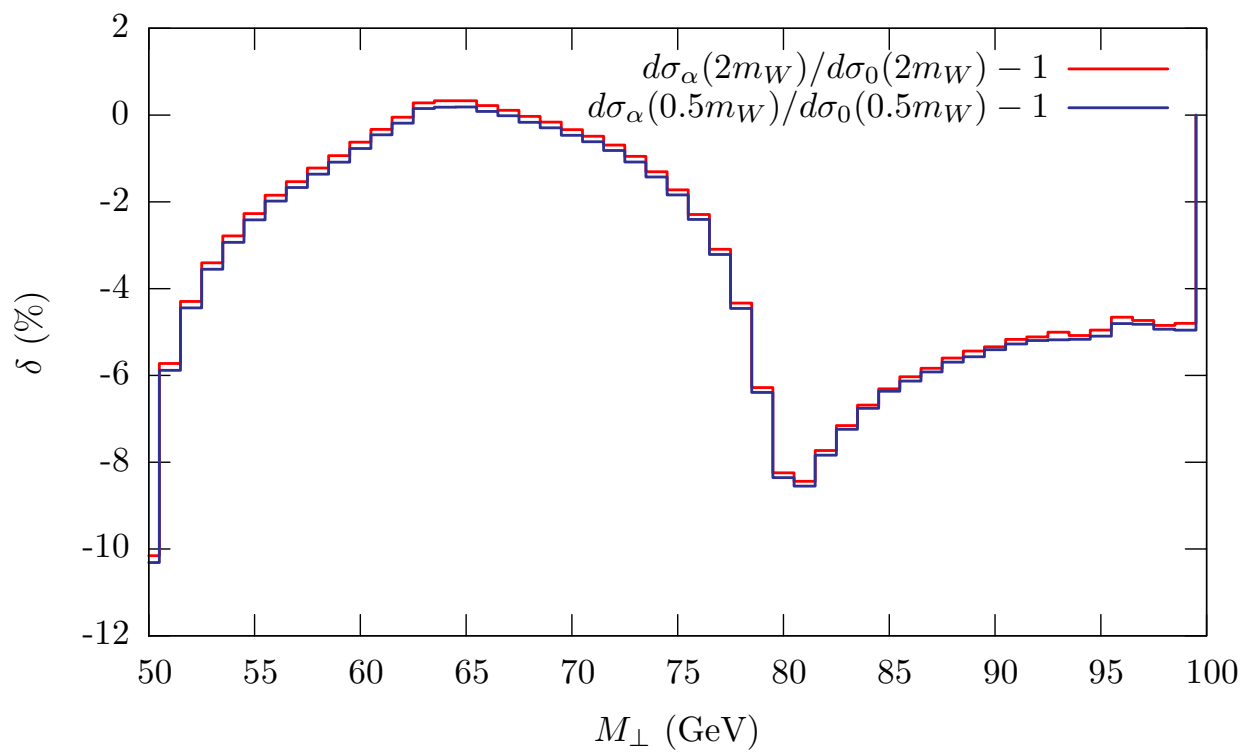

Figure 9: Relative effect, with respect to the corresponding Born, of the $\mathcal{O}(\alpha)$ corrections, computed with the factorization scale $M=m_{W} / 2$ and $M=2 m_{W}$.

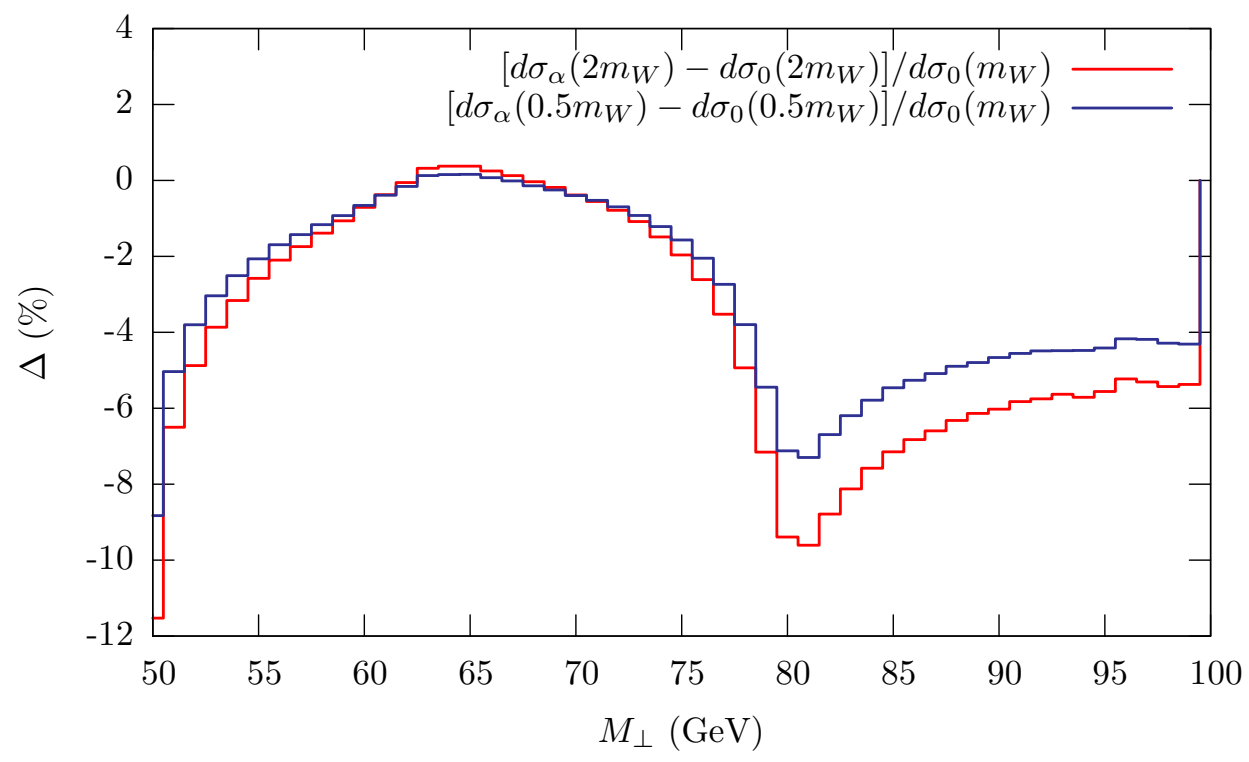

Figure 10: Relative effect of the $\mathcal{O}(\alpha)$ corrections, computed with the factorization scale $M=$ $m_{W} / 2$ and $M=2 m_{W}$, expressed in units of the Born cross section with $M=m_{W}$.

relative corrections:

$$
\delta(M) \equiv \frac{\sigma_{\alpha}(M)}{\sigma_{0}(M)}-1, \quad \Delta(M) \equiv \frac{\sigma_{\alpha}(M)-\sigma_{0}(M)}{\sigma_{0}\left(m_{W}\right)}
$$

In figure 9 we plot, for the transverse mass distribution, $\delta\left(0.5 m_{W}\right)$ and $\delta\left(2 m_{W}\right)$. The difference between the two curves can be interpreted as mainly due to the dependence of 
the $\mathcal{O}(\alpha)$ cross section on the choice of the QED factorization scale. We observe a variation at the per mille level of the transverse mass distribution, as already remarked in ref. [13].

In figure 10 we plot, for the transverse mass distribution, $\Delta\left(0.5 m_{W}\right)$ and $\Delta\left(2 m_{W}\right)$. The difference between the two curves can be interpreted as an estimate of the uncertainty due to missing $\mathcal{O}\left(\alpha \alpha_{s}\right)$ corrections, which are of the order of $1 \%$ around the $W$ resonance. In fact the numerator in $\Delta(M)$ is proportional to the hadron-level $\mathcal{O}(\alpha)$ corrections, which are a QCD-LO result. A change of the scale in the PDFs gives an estimate of the QCDNLO $\mathcal{O}\left(\alpha \alpha_{s}\right)$ effects $^{\dagger}$. We plot in figure 10 the $\mathcal{O}(\alpha)$ correction at two different scales, normalized to the Born cross section evaluated at a fixed scale $M=m_{W}$. In fact it is known that the introduction of QCD corrections stabilizes the total cross section against scale variations and we mimic this effect by setting $M=m_{W}$ in the denominator of $\Delta(M)$. In this way the uncertainty due to missing $\mathcal{O}\left(\alpha \alpha_{s}\right)$ corrections can be disentangled from other purely QCD effects.

To conclude this Section, we would like to comment on the possible relevance of the different effects and uncertainties on the $W$ boson mass measurement. It is known in the literature [2, 14, 20] that the distortion of the $M_{\perp}$ distribution around the peak due to the EW $\mathcal{O}(\alpha)$ and QED higher-order final-state corrections induces a shift of the extracted $m_{W}$ of the order of 100 and $10 \mathrm{MeV}$ respectively (for the muon case). In the new version of the generator HORACE both effects are included in a unique tool.

We remark that the theoretical uncertainty due to the QCD factorization scale choice, shown in figure 10, modifies the impact of the EW radiative corrections by an amount which can be of the order of $1 \%$ : these effects may induce a systematic error in the $m_{W}$ measurement which can be comparable with the aimed experimental accuracy and should be carefully considered in future experimental analyses.

\subsection{Rapidity distributions and charge asymmetry}

In figure 11 the muon and electron pseudo-rapidity distribution is presented in the approximations 1., 4. and 5. of table 1. We observe that the effect of the radiative corrections is almost constant over the whole range in pseudo-rapidity and that it is dominated by the $\mathcal{O}(\alpha)$ term, which gives a correction negative of approximately $-2.7 \%$ in the case of muons and of $-1.8 \%$ for recombined electrons. Higher-order terms modify the result at the per mille level.

In figure 12 the $W$-boson rapidity is also presented. With the chosen cuts, this distribution is essentially flat in the central rapidity interval $\left|y_{W}\right|<1.7$. The radiative corrections are negative and quite flat and reduce the Born distribution of about $-2 \%$ for the electrons and of $-3 \%$ for the muons, as shown in the inset.

The $W$ charge asymmetry presented in figure 13 is defined as

$$
A\left(\eta_{\ell}\right)=\frac{d \sigma^{+} / d \eta_{\ell}-d \sigma^{-} / d \eta_{\ell}}{d \sigma^{+} / d \eta_{\ell}+d \sigma^{-} / d \eta_{\ell}}
$$

\footnotetext{
${ }^{\dagger}$ We are not considering in this analysis other contributions of the same perturbative order, like the one due to the QED PDFs evolution multiplied with the diagrammatic QCD NLO corrections, because they are numerically suppressed.
} 


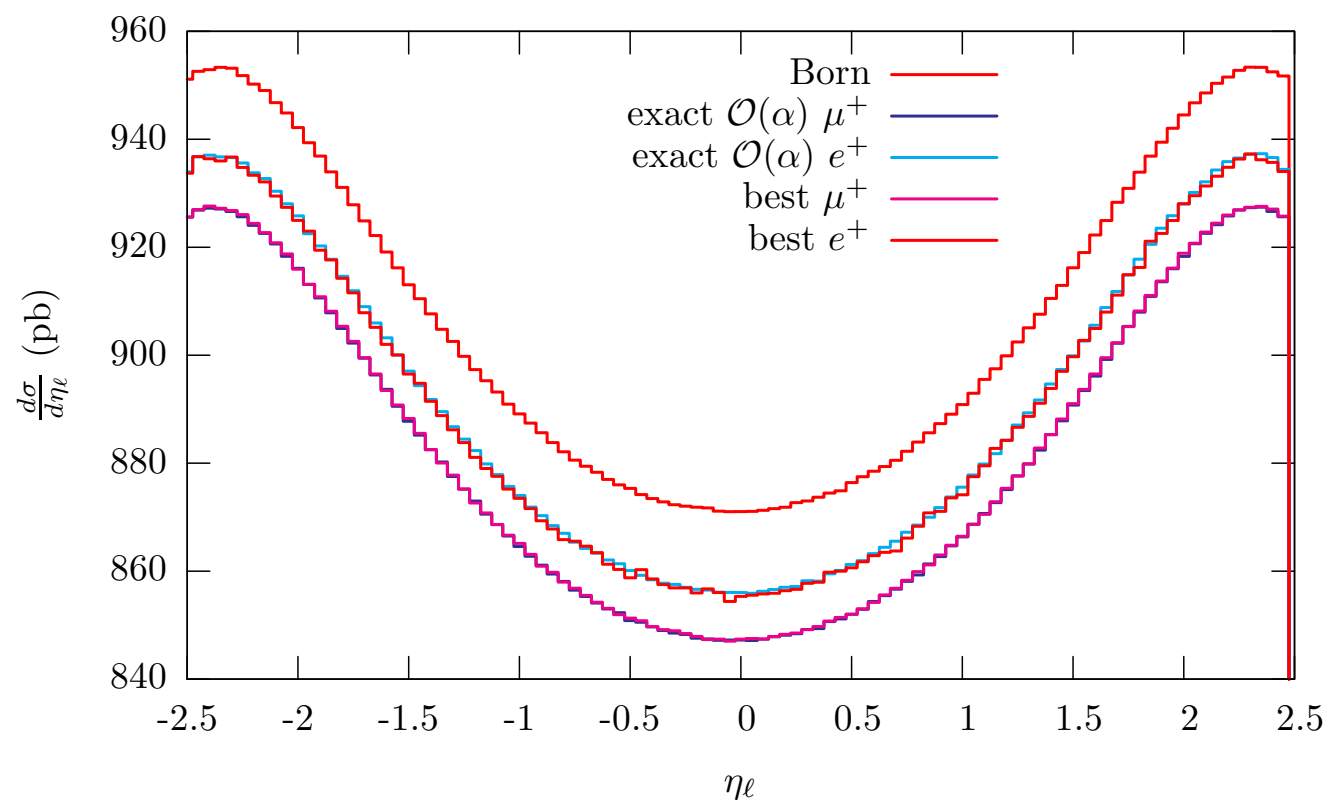

Figure 11: Charged lepton pseudo-rapidity distribution in Born, $\mathcal{O}(\alpha)$ and best approximations.

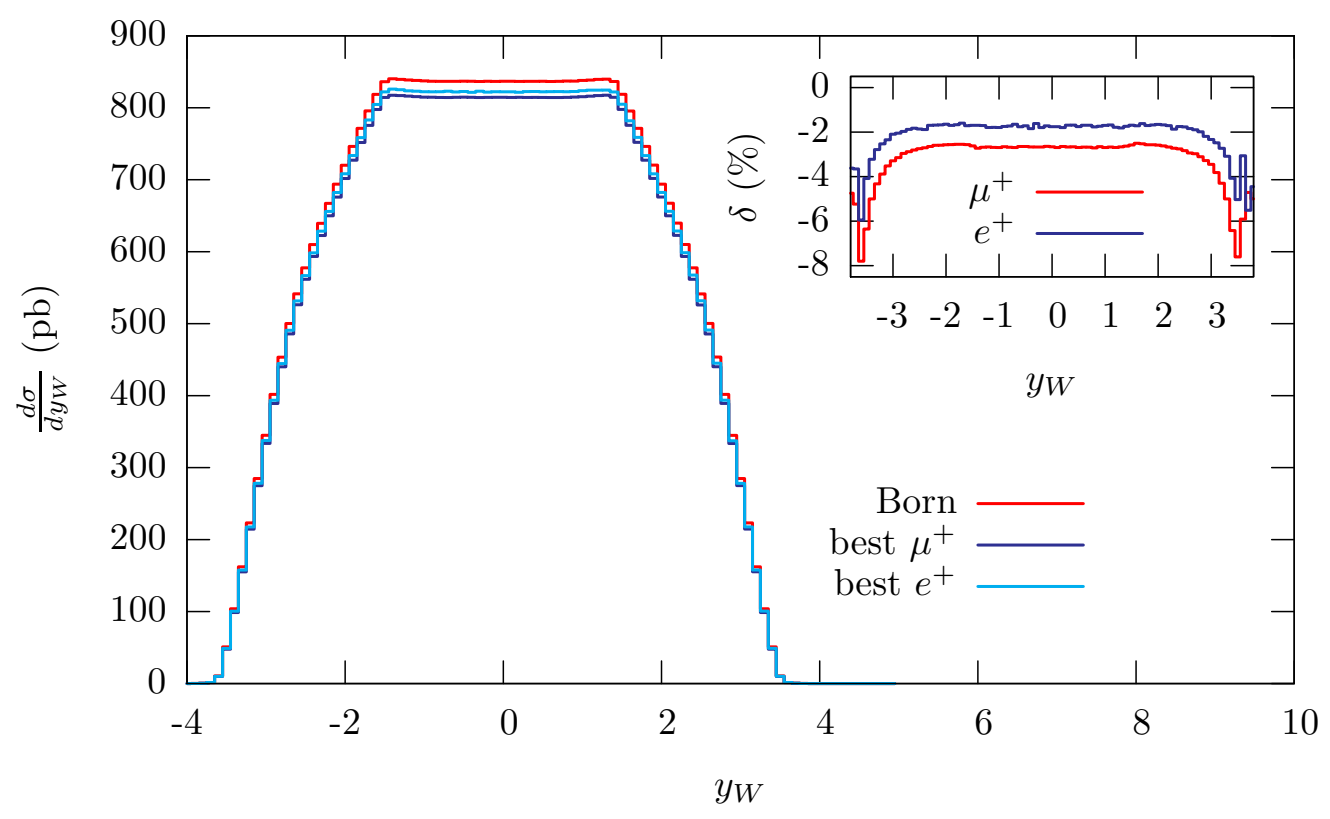

Figure 12: $W$ boson rapidity distribution in Born, $\mathcal{O}(\alpha)$ and best approximations.

where $d \sigma^{ \pm}=d \sigma\left(p \stackrel{(-)}{p} \rightarrow \ell^{ \pm} \nu+X\right)$; the asymmetry is due to the partonic content of the incoming hadrons, which leads to different lepton pseudo-rapidity distributions in the production of $W^{+}$or $W^{-}$. The charge asymmetry can be studied both at the Tevatron and at the LHC, with different results due to the two different initial states and to the different ranges of partonic $x$ probed at the two colliders. At the LHC the function is even under 

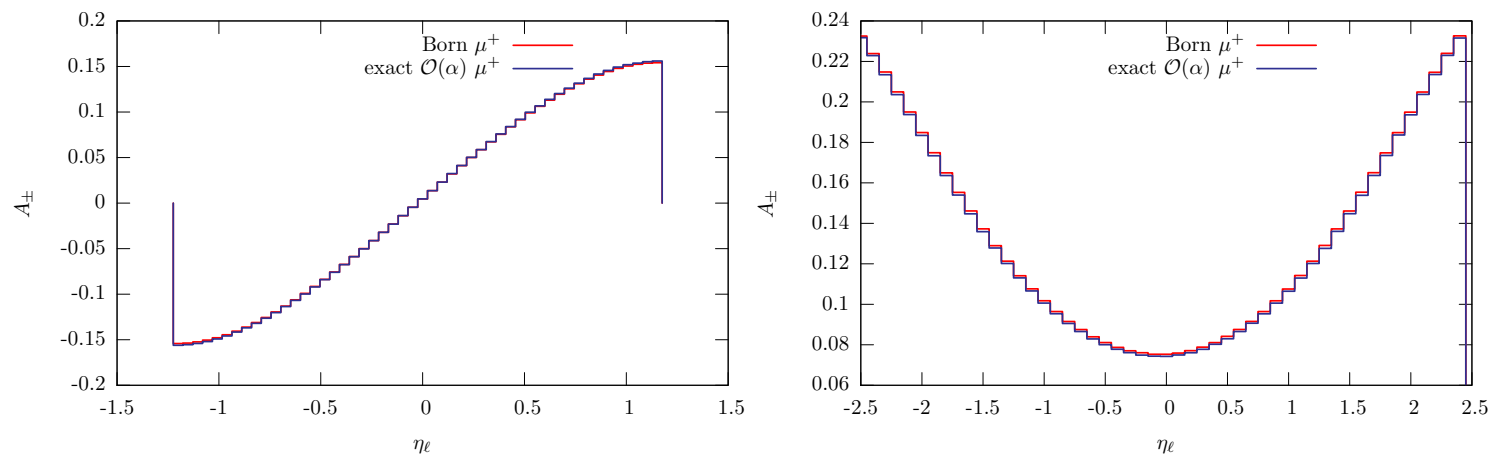

Figure 13: Charge asymmetry as function of the muon pseudo-rapidity at Tevatron $(\sqrt{s}=1.96$ $\mathrm{TeV})$ (left panel) and LHC $(\sqrt{s}=14 \mathrm{TeV})$ (right panel), in Born and $\mathcal{O}(\alpha)$ approximation.

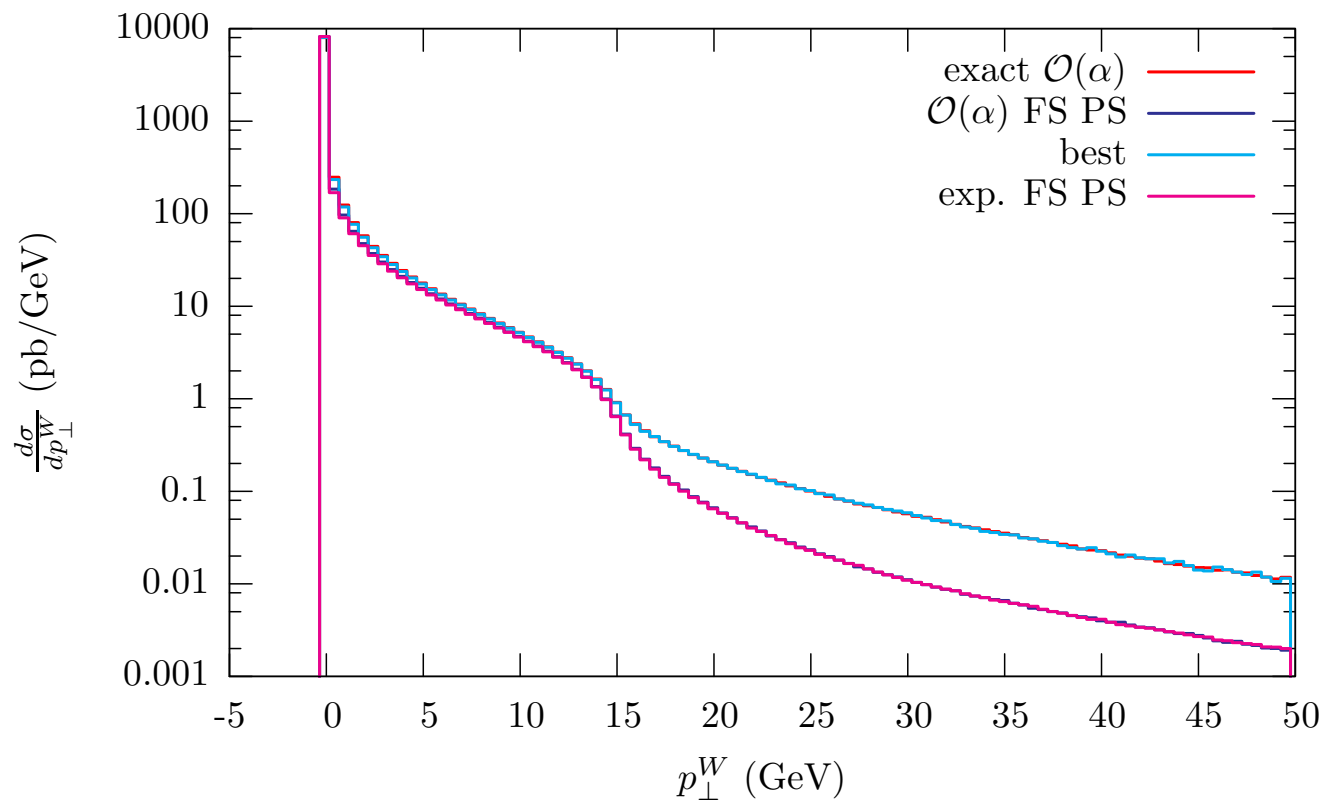

Figure 14: Distribution of the $W$ boson transverse momentum, defined as the transverse momentum of the final state lepton pair.

$\eta_{\ell} \rightarrow-\eta_{\ell}$, whereas it is odd at the Tevatron. The effect of the $\mathcal{O}(\alpha)$ corrections is at the $1 \%$ level, while higher-order effects are numerically negligible.

\section{4 $W$ transverse momentum and photonic observables}

Real photon radiation gives to the final state lepton pair a transverse momentum, which defines the $W$ boson transverse momentum, whose distribution is presented in figure 14 in the approximations 2., 3., 4. and 5. of table $1{ }^{\ddagger}$. The large difference in the tail is

\footnotetext{
${ }^{\ddagger}$ In the present study, the transverse motion of the $W$ boson, as due to initial-state QCD radiation, is neglected, because it requires a careful inclusion of QCD corrections, which is beyond the scope of the paper.
} 


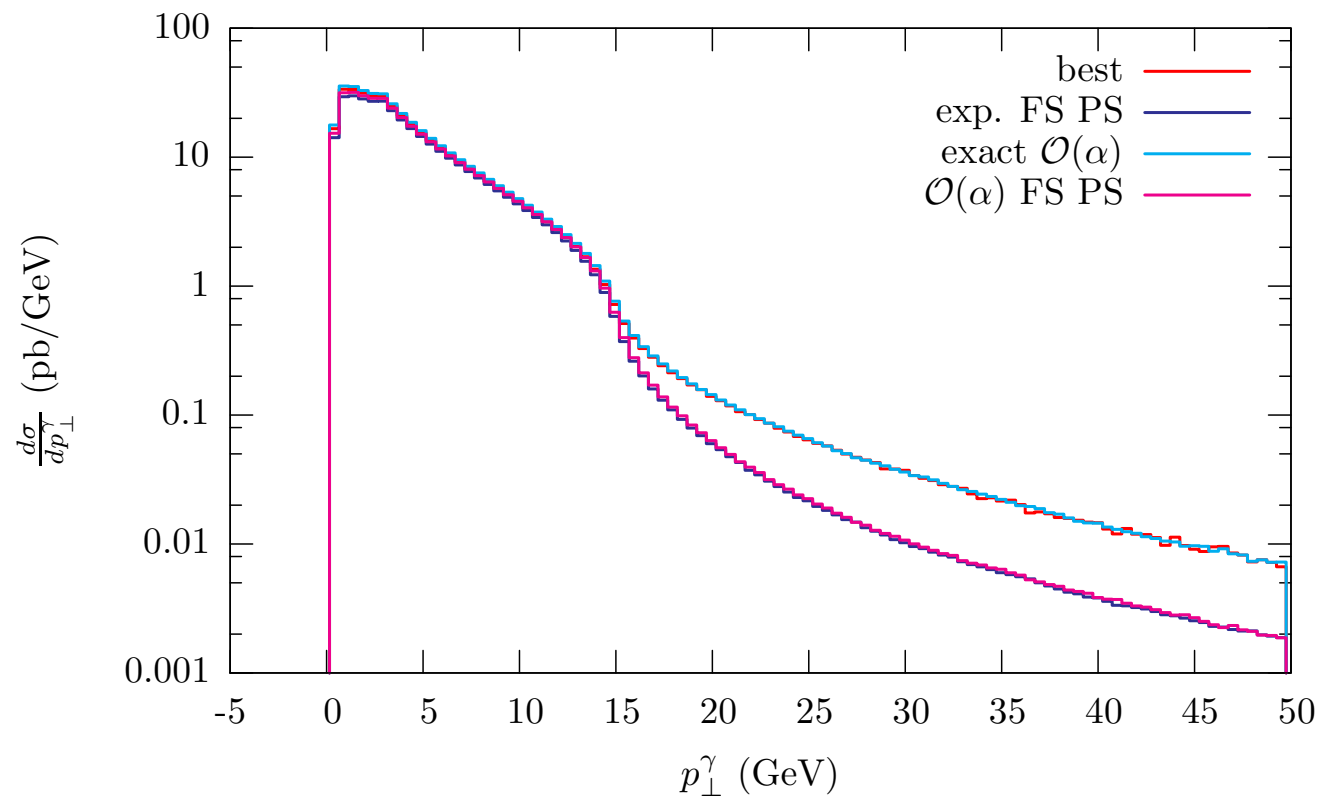

Figure 15: Distribution of the hardest photon transverse momentum.

due to a better description of the hard photon radiation given by the exact $\mathcal{O}(\alpha)$ matrix element, with respect to its Parton Shower approximation. The same comment applies when including, in the two cases, multiple-photon radiation.

We now present some distributions for the radiative Drell-Yan event, with a hard photon associated to the large transverse momentum lepton pair. This signature can be a source of information to study for instance the trilinear gauge boson $W W \gamma$ vertex [45]. We select the events by imposing the cuts of eq. (5.1) and requiring that the most energetic photon is detected, i.e. with rapidity $\left|\eta_{\gamma}\right|<2.5$ and $E_{\gamma}>3 \mathrm{GeV}$. The transverse momentum and the rapidity distributions of the hardest photon are plotted in figures 15 and 16. As in the $W$ transverse momentum case, the large difference in the tail in figure 15 is due to the better description of the hard photon radiation given by the exact $\mathcal{O}(\alpha)$ matrix element. Concerning the hardest photon rapidity distribution in figure 16, we compare first the lowest order results, which have been obtained in two different approximations, namely 2. and 3. of table 1 and observe the increase of the cross section given by the exact matrix element. When including multiple-photon radiation, the lowest order results receive a negative correction.

\section{Conclusions}

In this paper we presented a precision calculation of the charged current Drell-Yan process, which includes both the exact $\mathrm{EW} \mathcal{O}(\alpha)$ and the leading-log multiple-photon corrections. In order to keep consistently under control the two effects, a matching algorithm between the fixed order calculation and a QED Parton Shower, avoiding the double counting of the leading-log corrections, has been devised and implemented. To our knowledge, the 


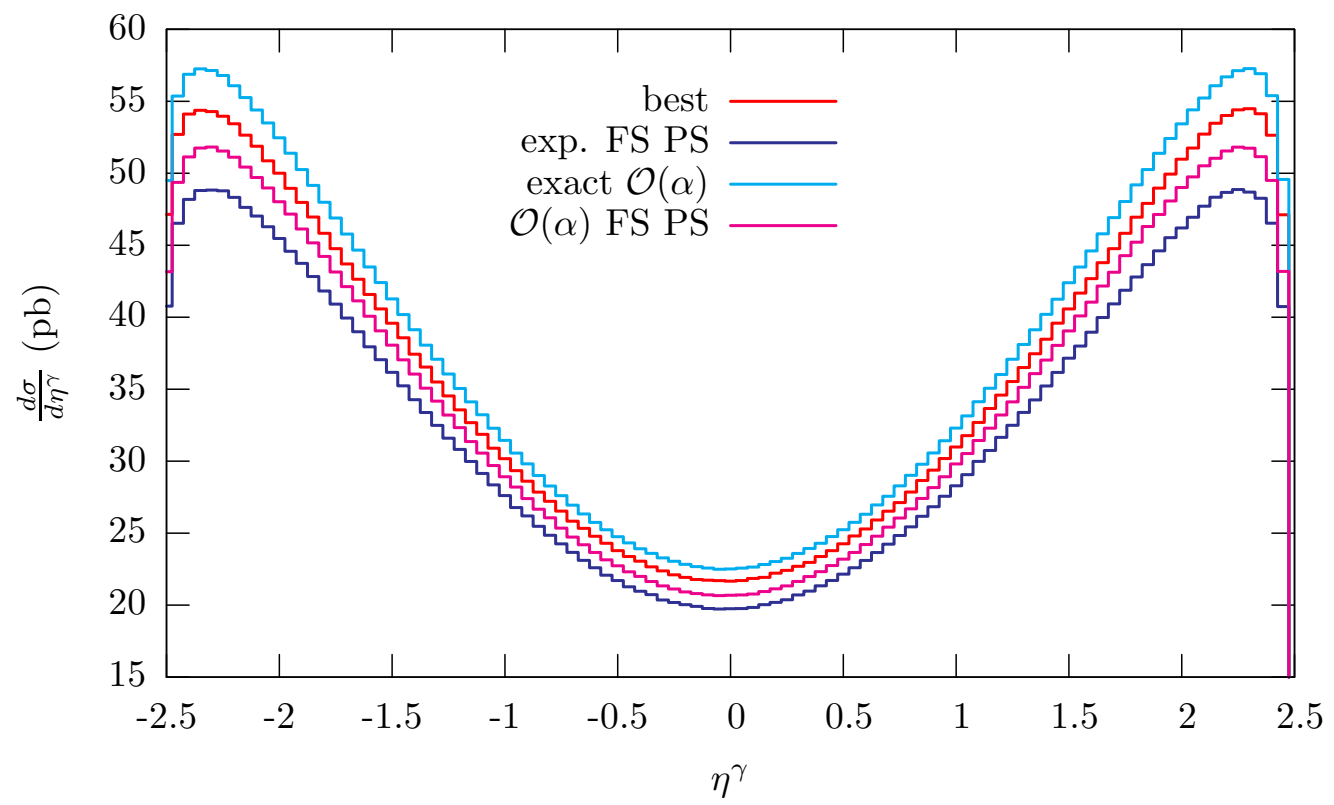

Figure 16: Distribution of the hardest photon pseudorapidity.

matching algorithm here presented is the first example of such an application in the field of EW radiative corrections. Initial-state QED collinear singularities have been regularized by means of finite quark masses, requiring a subtraction of the initial-state logarithms (in analogy to QCD NLO calculations) which are already accounted for in the evolution of the PDFs. The subtraction procedure, already known at $\mathcal{O}(\alpha)$, has been generalized to the QED resummed cross section, to systematically remove initial-state logarithms at all orders. Our results have been verified to be completely independent of the value of the quark masses, as expected.

We studied, with the new version of the event generator HORACE, the impact of different classes of radiative corrections on several physical observables, showing the importance of combining in a unique tool fixed order results with the resummation of multiple-photon radiation. In fact, radiative corrections induce effects ranging from several per mille to few per cent of the lowest-order cross section; they change the integrated cross section and the shape of the distributions.

For example, the value of the $W$ boson mass, extracted from the transverse mass distribution, is shifted, in the case of a final-state muon, by $\mathrm{EW} \mathcal{O}(\alpha)$ corrections of $\mathcal{O}(100 \mathrm{MeV})$ and of $\mathcal{O}(10 \mathrm{MeV})$ by multiple photon emission. These effects are important in view of the measurement foreseen at the LHC and Tevatron with an accuracy of $\Delta m_{W} \approx$ 15 and $\approx 30 \mathrm{MeV}$ and can now be included in a systematic way in the analysis of the experimental data. Far from the Jacobian peak, the tail of the transverse mass distribution, where the Drell-Yan process is a background to new gauge boson searches, receives large negative corrections due to the presence of the $\mathcal{O}(\alpha)$ EW Sudakov logarithms, of the order of $15-30 \%$. The effect of EW corrections is important also for the estimate of the detector 
acceptances in view of exploiting the $W$ production process as a precise luminosity monitor at the LHC, with an accuracy of some per cent.

We also studied radiative events, where at least one hard photon accompanies the final state lepton pair, which are a useful source of information for instance to measure the trilinear gauge boson vertex. In view of precision studies, the effects of the radiative corrections and of treating with the exact matrix element the photon radiation are sizeable on observables like the photon transverse momentum and rapidity distributions.

Besides the EW $\mathcal{O}(\alpha)$ and multiple-photon corrections, we studied some of the remaining theoretical uncertainties. We considered the ones due to the EW input scheme choice and to the QCD factorization scale choice. We would like to remark that they should be considered in detail to quantify, for example, the systematic error on the $W$ mass measurement.

A possible development of the work presented in this paper is the implementation in HORACE of the complete EW $\mathcal{O}(\alpha)$ corrections to the $Z$ production process and a detailed phenomenological study of their impact on the neutral current Drell-Yan process, which is important for many measurements and calibrations at hadron colliders. Furthermore, we are now working to combine the results presented here with QCD corrections, aiming at providing a unified tool which includes the relevant EW and QCD effects and which can be very useful for the experimental collaborations.

\section{Acknowledgments}

We are grateful to Andreij Arbuzov, Dima Bardin, Ulrich Baur, Stefan Dittmaier, Michael Krämer and Doreen Wackeroth for their precious collaboration during the tuned comparisons of the 2005 Les Houches workshop "Physics at TeV colliders". We are indebted with Mauro Moretti for his help in the comparison with the results of the ALPHA code and with Massimiliano Bellomo, Fulvio Piccinini and Giacomo Polesello for useful discussions and interest in our work. We thank Stefano Forte for a careful reading of the preliminary manuscript.

\section{References}

[1] S. D. Drell and T. M. Yan, Phys. Rev. Lett. 25 (1970) 316 [Erratum-ibid. 25 (1970) 902].

[2] V. M. Abazov et al. [CDF Collaboration], Phys. Rev. D 70 (2004) 092008 [arXiv:hep-ex/0311039];

A. Abulencia et al. [CDF Collaboration], [arXiv:hep-ex/0508029].

[3] H. L. Lai et al., Phys. Rev. D 51 (1995) 4763 [arXiv:hep-ph/9410404];

A. D. Martin, R. G. Roberts, W. J. Stirling and R. S. Thorne, Eur. Phys. J. C 28 (2003) 455 [arXiv:hep-ph/0211080].

[4] M. Dittmar, F. Pauss and D. Zurcher, Phys. Rev. D 56, 7284 (1997) [arXiv:hep-ex/9705004].

[5] S. Frixione and M. L. Mangano, JHEP 0405, 056 (2004) [arXiv:hep-ph/0405130].

[6] F. Abe et al. [CDF Collaboration], Phys. Rev. Lett. 79 (1997) 2192. 
[7] G. Altarelli, R. K. Ellis and G. Martinelli, Nucl. Phys. B 157 (1979) 461.

[8] R. Hamberg, W. L. van Neerven and T. Matsuura, Nucl. Phys. B 359, 343 (1991) [Erratum-ibid. B 644, 403 (2002)].

[9] C. Anastasiou, L. J. Dixon, K. Melnikov and F. Petriello, Phys. Rev. Lett. 91, 182002 (2003) [arXiv:hep-ph/0306192];

C. Anastasiou, L. J. Dixon, K. Melnikov and F. Petriello, Phys. Rev. D 69, 094008 (2004) [arXiv:hep-ph/0312266];

K. Melnikov and F. Petriello, "Electroweak gauge boson production at hadron colliders through $\mathcal{O}\left(\right.$ alpha $\left.a_{s}^{2}\right)$ ", [arXiv:hep-ph/0609070].

[10] D. Wackeroth and W. Hollik, Phys. Rev. D 55 (1997) 6788 [arXiv:hep-ph/9606398].

[11] U. Baur, S. Keller and D. Wackeroth, Phys. Rev. D 59 (1999) 013002 [arXiv:hep-ph/9807417].

[12] V. A. Zykunov, Eur. Phys. J. directC 3 (2001) 9 [arXiv:hep-ph/0107059]; V. A. Zykunov, "Weak radiative corrections to Drell-Yan process for large invariant mass of di-lepton pair", [arXiv:hep-ph/0509315].

[13] S. Dittmaier and M. Krämer, Phys. Rev. D 65 (2002) 073007 [arXiv:hep-ph/0109062].

[14] U. Baur and D. Wackeroth, Phys. Rev. D 70 (2004) 073015 [arXiv:hep-ph/0405191].

[15] A. Arbuzov, D. Bardin, S. Bondarenko, P. Christova, L. Kalinovskaya, G. Nanava and R. Sadykov, Eur. Phys. J. C 46 (2006) 407 [arXiv:hep-ph/0506110].

[16] G. Corcella et al., "HERWIG 6.5 release note", [arXiv:hep-ph/0210213].

[17] T. Sjostrand, P. Eden, C. Friberg, L. Lonnblad, G. Miu, S. Mrenna and E. Norrbin, Comput. Phys. Commun. 135 (2001) 238 [arXiv:hep-ph/0010017].

[18] S. Frixione and B. R. Webber, JHEP 0206 (2002) 029 [arXiv:hep-ph/0204244].

[19] C. Balazs and C. P. Yuan, Phys. Rev. D 56 (1997) 5558 [arXiv:hep-ph/9704258].

[20] C. M. Carloni Calame, G. Montagna, O. Nicrosini and M. Treccani, Phys. Rev. D 69 (2004) 037301 [arXiv:hep-ph/0303102].

[21] http://www.pv.infn.it/hepcomplex/horace.html

[22] C. M. Carloni Calame, C. Lunardini, G. Montagna, O. Nicrosini and F. Piccinini, Nucl. Phys. B 584, 459 (2000) [arXiv:hep-ph/0003268];

C. M. Carloni Calame, Phys. Lett. B 520 (2001) 16 [arXiv:hep-ph/0103117].

[23] W. Placzek and S. Jadach, Eur. Phys. J. C 29 (2003) 325 [arXiv:hep-ph/0302065].

[24] D. R. Yennie, S. C. Frautschi and H. Suura, Ann. Phys.13 (1961) 379.

[25] P. Golonka and Z. Was, Eur. Phys. J. C 45 (2006) 97 [arXiv:hep-ph/0506026].

[26] Q. H. Cao and C. P. Yuan, Phys. Rev. Lett. 93 (2004) 042001 [arXiv:hep-ph/0401026].

[27] P. Golonka and Z. Was, Eur. Phys. J. C 45 (2006) 97 [arXiv:hep-ph/0506026];

C. M. Carloni Calame, S. Jadach, G. Montagna, O. Nicrosini and W. Placzek, Acta Phys. Polon. B 35, 1643 (2004) [arXiv:hep-ph/0402235].

[28] C. Buttar et al., [arXiv:hep-ph/0604120]. 
[29] See, for example, Z. Nagy and D.E. Soper, "QCD and Monte Carlo generators", [arXiv:hep-ph/0607046], and references therein.

[30] G. Balossini, C. M. C. Calame, G. Montagna, O. Nicrosini and F. Piccinini, "Matching perturbative and Parton Shower corrections to Bhabha process at flavour factories", [arXiv:hep-ph/0607181].

[31] T. Hahn, Comput. Phys. Commun. 140 (2001) 418 [arXiv:hep-ph/0012260].

[32] T. Hahn and M. Perez-Victoria, Comput. Phys. Commun. 118 (1999) 153 [arXiv:hep-ph/9807565].

[33] G. J. van Oldenborgh and J. A. M. Vermaseren, Z. Phys. C 46, 425 (1990).

[34] L. F. Abbott, Nucl. Phys. B 185, 189 (1981);

A. Denner, G. Weiglein and S. Dittmaier, Nucl. Phys. B 440 (1995) 95 [arXiv:hep-ph/9410338].

[35] F. Jegerlehner, J. Phys. G 29 (2003) 101 [arXiv:hep-ph/0104304].

[36] A. Sirlin, Phys. Rev. D 22 (1980) 971.

[37] A. Denner, Fortsch. Phys. 41 (1993) 307.

[38] J. A. M. Vermaseren, "New features of FORM", [arXiv:math-ph/0010025].

[39] F. Caravaglios and M. Moretti, Phys. Lett. B 358 (1995) 332 [arXiv:hep-ph/9507237].

[40] See, for example, M. E. Peskin and D. V. Schroeder, "An introduction to Quantum Field Theory", Addison-Wesley, 1995.

[41] A. D. Martin, R. G. Roberts, W. J. Stirling and R. S. Thorne, Eur. Phys. J. C 39 (2005) 155 [arXiv:hep-ph/0411040].

[42] T. Hahn, Comput. Phys. Commun. 168 (2005) 78 [arXiv:hep-ph/0404043].

[43] H. Spiesberger, Phys. Rev. D 52 (1995) 4936 [arXiv:hep-ph/9412286].

[44] M. Roth and S. Weinzierl, Phys. Lett. B 90 (2004) 190 [arXiv:hep-ph/0403200].

[45] F. Abe et al. [CDF Collaboration], Phys. Rev. Lett. 74 (1995) 1936;

U. Baur and D. Zeppenfeld, Nucl. Phys. B 308, 127 (1988). 